\title{
Variational Integrators and the Newmark Algorithm for Conservative and Dissipative Mechanical Systems
}

\author{
C. Kane \\ Graduate Aeronautical Laboratories and CDS \\ California Institute of Technology \\ Pasadena, CA 91125 \\ kane@cds.caltech.edu \\ J. E. Marsden \\ CDS 107-81 \\ California Institute of Technology \\ Pasadena, CA 91125 \\ marsden@cds.caltech.edu \\ M. Ortiz \\ Graduate Aeronautical Laboratories \\ California Institute of Technology \\ Pasadena, CA 91125 \\ ortiz@madrid.caltech.edu \\ M. West \\ CDS 107-81 \\ California Institute of Technology \\ Pasadena, CA 91125 \\ mwest@cds.caltech.edu
}

March, 1999, This version: November 25, 1999 


\begin{abstract}
The purpose of this work is twofold. First, we demonstrate analytically that the classical Newmark family as well as related integration algorithms are variational in the sense of the Veselov formulation of discrete mechanics. Such variational algorithms are well known to be symplectic and momentum preserving and to often have excellent global energy behavior. This analytical result is verified through numerical examples and is believed to be one of the primary reasons that this class of algorithms performs so well.

Second, we develop algorithms for mechanical systems with forcing, and in particular, for dissipative systems. In this case, we develop integrators that are based on a discretization of the Lagrange d'Alembert principle as well as on a variational formulation of dissipation. It is demonstrated that these types of structured integrators have good numerical behavior in terms of obtaining the correct amounts by which the energy changes over the integration run.
\end{abstract}

\title{
Contents
}

1 Introduction and Background 3

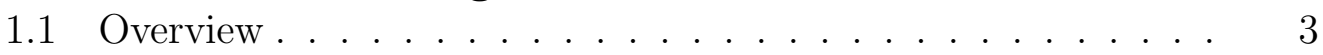

1.2 Background on Geometric Integrators . . . . . . . . . . . . . 4

2 Variational Integrators $\quad 6$

2.1 A Review of Variational Integrators . . . . . . . . . . . . . . . 6

2.2 Construction of Mechanical Integrators . . . . . . . . . . . . 10

2.3 Representations of Variational Integrators . . . . . . . . . . . 11

3 The Newmark Algorithm is Variational 13

3.1 Newmark Schemes . . . . . . . . . . . . . . . . . 13

3.2 Central Differences and Linear Systems . . . . . . . . . . . . . 15

3.3 Shadowing of Newmark Trajectories . . . . . . . . . . . . . . . 16

3.4 Newmark Itself is Variational . . . . . . . . . . . . . . . . . 19

3.5 Consequences of Newmark's Variational Nature . . . . . . . . 21

3.6 Minimization Structure of the Newmark Algorithm . . . . . . 23

4 Numerical Tests for Conservative Systems 25

4.1 Example System . . . . . . . . . . . . . . . . . 25

4.2 Tested Algorithms . . . . . . . . . . . . . . . . . . 25

4.3 Results . . . . . . . . . . . . . . . 26 
5 Numerical Algorithms for Systems with Friction 28

5.1 Discrete Lagrange-d'Alembert Principle . . . . . . . . . . . 29

5.2 The Newmark Algorithm with Forcing is Variational . . . . . 30

5.3 A Two-Step Variational Principle for Friction . . . . . . . . 32

5.4 Minimization Structure of Newmark with Friction. . . . . . . . 33

6 Numerical Tests for Dissipative Systems 34

6.1 Example System . . . . . . . . . . . . . . . . . . . . . 34

6.2 Tested Algorithms . . . . . . . . . . . . . . . . . . . 34

6.3 Results . . . . . . . . . . . . . . . . . . . 35

$\begin{array}{lll}7 & \text { Conclusions and Future Work } & 37\end{array}$

\section{Introduction and Background}

\subsection{Overview}

We begin with the following intriguing quote from Simo, Tarnow and Wong [1992]:

What may seem surprising is that all of the implicit members of the Newmark family, perhaps the most widely used time-stepping algorithms in nonlinear structural dynamics, are not designed to conserve energy and also fail to conserve momentum. Among the explicit members, only the central difference method preserves momentum.

Rather little has been done on the analysis and structure of the Newmark family since this work of Simo, Tarnow and Wong. The present paper aims to fill this gap. The key to our approach is the recent progress in variational integrators based on the Veselov theory of discrete mechanics and the inclusion of dissipation and forcing into these schemes using optimization techniques.

We shall show (in a sense that is not entirely obvious) that the classical Newmark scheme is indeed variational and so is symplectic and momentum preserving. This appears, at first sight, to contradict the above quote. This apparent paradox is resolved by realizing that the construction of the conserved symplectic form and the momentum is not done in a completely obvious way, and thus it is a non-canonical symplectic form and non-standard momenta that are conserved.

It is known that symplectic integrators often have remarkable near energy preserving properties and we believe that the symplectic nature of the Newmark scheme goes a long way towards explaining their excellent performance, 
often better than that of high order schemes for moderately long time integrations of conservative and forced systems (see, for example, Hairer and Lubich [1997] and Reich [1999] and references therein). We shall present specific examples of this phenomenon in this paper.

Main Results of this Paper. The main accomplishments of the present paper are:

1. We show the precise sense in which the Newmark algorithm is variational.

2. As a consequence of its variational nature, the Newmark scheme exactly preserves a certain symplectic structure and a certain algorithmically computed momentum (linear or angular momentum, as appropriate).

3. Dissipation and forcing are incorporated into the Newmark and variational schemes

4. Numerical tests of these schemes are given for some relatively simple systems to demonstrate their effective performance.

\subsection{Background on Geometric Integrators}

Mechanical Integrators. There is a large literature that has developed on the use of energy-momentum and symplectic-momentum integrators. For time stepping algorithms with fixed time steps, the theorem of Ge and Marsden [1988] led to a general division of algorithms into those that are energymomentum preserving and those that are symplectic-momentum preserving.

If one takes a spacetime view of variational integrators, as is advocated in Marsden, Patrick and Shkoller [1998], then one can have integrators that preserve the energy, momentum and the symplectic structure, as has been shown in Kane, Marsden and Ortiz [1999]. Papers typified by Simo and Tarnow [1992], Simo, Tarnow, and Wong [1992] and Gonzalez [1996] have focussed on energy preserving algorithms, but they presumably fail (except, perhaps, in special cases, such as integrable systems) to be symplectic. For a survey of other literature, see McLachlan and Scovel [1996].

Accuracy of Solutions. It is well known that structure preservation alone does not guarantee accuracy of individual trajectories. (See, e.g., Ortiz [1986] and Simo and Gonzalez [1993]). Complicating this issue is the fact that for systems with complicated, unstable, or chaotic trajectories, accuracy of individual trajectories is presumably not the correct question to ask. Rather one should probably concentrate on accurate prediction of statistical or stably reproducible properties of solution families. However, as has been frequently 
demonstrated and we shall do so here as well, in many circumstances structure preserving algorithms often perform remarkably well-far better than an error analysis would suggest.

One also must be cautious here. When the time steps are too large, symplectic schemes may have bad energy behavior and fail to be accurate. See, for example, Gonzalez and Simo [1996].

Discrete Lagrangian Mechanics and Integrators. Veselov [1988] developed a discrete version of Lagrangian mechanics with an emphasis on variational methods (see also Moser and Veselov [1991]). In particular, he showed, as in the theory of generating functions, that the corresponding discrete EulerLagrange equations define symplectic maps. Using these ideas, it can be shown that several well known algorithms, such as the Verlet and shake methods, are variational integrators (see MacKay [1992] and Wendlandt and Marsden [1997]). There have been considerable additional efforts in this area, such as Marsden, Patrick and Shkoller [1998], Kane, Marsden and Ortiz [1999], Marsden, Pekarsky and Shkoller [1999a,b] Bobenko, Lorbeer, and Suris [1998], and Bobenko and Suris [1999]. We shall comment on some of the related developments below and in the body of the paper.

In structural mechanics, the $\beta=0, \gamma=1 / 2$ (central differences) member of the widely used Newmark family has been known to be symplectic and momentum preserving for some time (see, for example, Simo, Tarnow, and Wong [1992]). This was shown by more or less ad hoc techniques as a "curious observation". One of our main results is to extend this to all members of the Newmark family (for unforced mechanical systems). We shall do this by showing that the Newmark algorithm is indeed variational in the sense of Veselov.

Dissipation. A second main point of this paper is to demonstrate the effectiveness of variational techniques for dissipative or more generally, forced mechanical systems. One possibility is that dissipative effects can be dealt with by means of product formulas, as in Armero and Simo [1992, 1993, 1996]. Another is to incorporate the dissipative effects into the variational principle, as in Ortiz and Stainier [1999] (see also Radovitzky and Ortiz [1999]). We shall abstract some of these techniques in this paper.

Constraints. Constraints are of obvious importance for integrators. We do not discuss these in any detail in this paper. However, we do mention that variational integrators usually handle constraints in a simple and efficient way using Lagrange multipliers, as shown in, for example, Wendlandt and Marsden [1997]. In addition, when handled variationally, constraints do not disturb the 
symplectic or conservative nature of the algorithms. It is well known that other techniques can run into trouble in this regard. See, for example, the discussion of this point in Leimkuhler and Reich [1994].

Multisymplectic Integrators. Variational methods also generalize to pde's using multisymplectic geometry with the result being a class of multisymplectic momentum integrators. See Marsden, Patrick, and Shkoller [1998] for details and numerical examples; see also Reich and Bridges [1999]. This type of approach should ultimately be of use in elastodynamics as well as ocean dynamics, for example.

Symmetry and Reduction. We should also mention that for mechanical systems with symmetry, the investigation of discrete versions of reduction theory, such as Euler-Poincaré reduction are of current interest (see, for example, Marsden, Pekarsky and Shkoller [1999a,b]). We will not be making use of this reduction theory in this paper, but this work is related since our integrators are intended to preserve symmetry.

\section{Variational Integrators}

\subsection{A Review of Variational Integrators}

Variational Algorithms. Variational schemes following the Veselov discretization technique are now well known and we briefly review them here. See, for example, MacKay [1992], Wendlandt and Marsden [1997], Marsden, Patrick and Shkoller [1998], Kane, Marsden and Ortiz [1999], and Marsden, Pekarsky and Shkoller [1999a,b].

These schemes are known to be automatically symplectic (and are often second order accurate) integrators that preserve conserved quantities (such as linear and angular momentum) associated with symmetries provided the discrete Lagrangian has these symmetries.

We briefly recall these schemes here. Given a configuration space $Q$, a discrete Lagrangian is a map

$$
L_{d}: Q \times Q \rightarrow \mathbb{R} .
$$

In practice, $L_{d}$ is obtained by approximating the action function associated with a given Lagrangian as we shall discuss later, but regard $L_{d}$ as given for the moment. The time step information will be contained in $L_{d}$ and we regard $L_{d}$ as a function of two nearby points $\left(q_{k}, q_{k+1}\right)$. 
Example. Consider a continuous Lagrangian of the standard form

$$
L(q, \dot{q})=\frac{1}{2} \dot{q}^{T} M \dot{q}-V(q),
$$

where $M$ is a symmetric positive definite mass matrix, $q \in \mathbb{R}^{n}=Q$ and $V$ is a given potential. Define an associated discrete Lagrangian, $L_{d}^{\alpha}: Q \times Q \rightarrow \mathbb{R}$ by

$$
L_{d}^{\alpha}\left(q_{0}, q_{1}\right)=h L\left((1-\alpha) q_{0}+\alpha q_{1}, \frac{q_{1}-q_{0}}{h}\right),
$$

where $h \in \mathbb{R}_{+}$is the time step and $0 \leq \alpha \leq 1$ is an interpolation parameter. Using the given form of $L$, this becomes

$$
L_{d}^{\alpha}\left(q_{0}, q_{1}\right)=h \frac{1}{2}\left(\frac{q_{1}-q_{0}}{h}\right)^{T} M\left(\frac{q_{1}-q_{0}}{h}\right)-h V\left((1-\alpha) q_{0}+\alpha q_{1}\right) .
$$

We shall return to a systematic study of discrete Lagrangians of this form shortly in $\S 2.2$.

For a positive integer $N$, the action sum is the map $S_{d}: Q^{N+1} \rightarrow \mathbb{R}$ defined by

$$
S_{d}=\sum_{k=0}^{N-1} L_{d}\left(q_{k}, q_{k+1}\right),
$$

where $q_{k} \in Q$ and $k$ is a nonnegative integer. The action sum is the discrete analog of the action integral

$$
S=\int_{a}^{b} L(q(t), \dot{q}(t)) d t .
$$

The discrete variational principle states that the evolution equations extremize the action sum given fixed end points, $q_{0}$ and $q_{N}$. Extremizing $S_{d}$ over $q_{1}, \cdots, q_{N-1}$ leads to the discrete Euler-Lagrange (DEL) equations:

$$
D_{1} L_{d}\left(q_{k+1}, q_{k+2}\right)+D_{2} L_{d}\left(q_{k}, q_{k+1}\right)=0
$$

for all $k=1, \cdots, N-1$, where $D_{1} L$ denotes the derivative of $L$ with respect to its first slot. We can write this equation in terms of a discrete algorithm:

$$
\Phi: Q \times Q \rightarrow Q \times Q
$$

defined implicitly by

$$
D_{1} L_{d} \circ \Phi+D_{2} L_{d}=0
$$

i.e.,

$$
\Phi\left(q_{k}, q_{k+1}\right)=\left(q_{k+1}, q_{k+2}\right) .
$$

If, for each fixed $q \in Q$, the map $D_{1} L_{d}(q, q): T_{q} Q \rightarrow T_{q}^{*} Q$ is invertible, then $D_{1} L_{d}: Q \times Q \rightarrow T^{*} Q$ is locally invertible in a neighborhood of the identity and so the algorithm $\Phi$ is well defined for small time steps. 
Example. For the discrete Lagrangian (2.2), the DEL equations are readily computed to be

$$
\begin{aligned}
& \frac{M}{h^{2}}\left(q_{k+2}-2 q_{k+1}+q_{k}\right) \\
& \quad=-(1-\alpha) \nabla V\left((1-\alpha) q_{k+1}+\alpha q_{k+2}\right)-\alpha \nabla V\left((1-\alpha) q_{k}+\alpha q_{k+1}\right) .
\end{aligned}
$$

This specific example will play an important role in subsequent sections of the paper.

Variational Algorithms are Symplectic. To explain the sense in which the algorithm is symplectic, first define the fiber derivative (or the discrete Legendre transform) by

$$
\mathbb{F} L_{d}: Q \times Q \rightarrow T^{*} Q ; \quad\left(q_{0}, q_{1}\right) \mapsto\left(q_{1}, D_{2} L_{d}\left(q_{0}, q_{1}\right)\right)
$$

and define the 2-form $\Omega_{L_{d}}$ on $Q \times Q$ by pulling back the canonical 2-form $\Omega=d q^{i} \wedge d p_{i}$ from $T^{*} Q$ to $Q \times Q$ :

$$
\Omega_{L_{d}}=\left(\mathbb{F} L_{d}\right)^{*}(\Omega)
$$

The alternative discrete fiber derivative $\tilde{\mathbb{F}} L_{d}\left(q_{0}, q_{1}\right):=\left(q_{0},-D_{1} L_{d}\left(q_{0}, q_{1}\right)\right)$ may be used and the results obtained will be essentially unchanged. Either definition may be regarded as an analog of the standard Legendre transform:

$$
\mathbb{F} L: T Q \rightarrow T^{*} Q ; \quad(q, \dot{q}) \mapsto\left(q, D_{2} L(q, \dot{q})\right) .
$$

An expression for $\Omega_{L_{d}}$ in terms of the coordinates $q_{0}^{i}, q_{1}^{i}$ of the points $q_{0}, q_{1}$ is

$$
\Omega_{L_{d}}=\frac{\partial^{2} L_{d}}{\partial q_{0}^{i} \partial q_{1}^{j}}\left(q_{0}, q_{1}\right) d q_{0}^{i} \wedge d q_{1}^{j} .
$$

A fundamental fact is that the algorithm $\Phi$ exactly preserves the symplectic form $\Omega_{L_{d}}$. That is, $\Phi^{*} \Omega_{L_{d}}=\Omega_{L_{d}}$.

One proof of this is to simply verify it with a straightforward calculationsee Wendlandt and Marsden [1997] for the details. Another approach is to derive the same conclusion directly from the variational structure, as is done in Marsden, Patrick, and Shkoller [1998]. This value of this latter approach is particularly apparent when one wishes to consider extensions to variable timestep schemes, as in Kane, Marsden and Ortiz [1999] and to problems involving collisions as in Kane, Repetto, Ortiz and Marsden [1999] and Kane, Marsden, Ortiz and Pandolfi [1999]. 
The Algorithm Preserves Momentum. Recall that Noether's theorem states that a continuous symmetry of the Lagrangian leads to conserved quantities, such as linear and angular momentum. One can directly derive these conservation laws using the invariance of the variational principle (this is the way Noether originally did it).

Assume that the discrete Lagrangian is invariant under the action of a Lie group $G$ on $Q$, and let $\xi \in \mathfrak{g}$, the Lie algebra of $G$. By analogy with the continuous case, define the discrete momentum map, $\mathbf{J}_{d}: Q \times Q \rightarrow \mathfrak{g}^{*}$ by

$$
\left\langle\mathbf{J}_{d}\left(q_{k}, q_{k+1}\right), \xi\right\rangle:=\left\langle D_{2} L_{d}\left(q_{k}, q_{k+1}\right), \xi_{Q}\left(q_{k+1}\right)\right\rangle .
$$

A second fundamental fact is that the algorithm $\Phi$ exactly preserves the momentum map.

Example. Consider a Lagrangian of the usual kinetic minus potential energy form as above:

$$
L(q, \dot{q})=\frac{1}{2} \dot{q}^{T} M \dot{q}-V(q)
$$

and the discrete Lagrangian (2.2). Assume that $V$ is independent of $q^{1}$ so that $p_{1}=[M \dot{q}]_{1}$ (the first component of $M \dot{q}$ ) is a constant of the motion for the continuous system ( $q^{1}$ is a cyclic variable.)

The corresponding conserved discrete momentum map given by (2.10) is the algorithmic analog of the momentum in the first coordinate direction (notice that the time step $h$ cancels in the calculation):

$$
J_{d}\left(q_{k}, q_{k+1}\right)=\left[M\left(q_{k+1}-q_{k}\right)\right]_{1} .
$$

Being a constant of the motion means that $J_{d}\left(q_{k}, q_{k+1}\right)$ is independent of $k$. One of course can verify this directly, but it is guaranteed by the theory. Related examples such as linear and angular momentum for systems of particles or rigid bodies proceed in a similar way.

Associated Energy. The energy function associated to a given discrete Lagrangian is defined, according to Kane, Marsden and Ortiz [1999], by

$$
E_{d}\left(q_{0}, q_{1}, h\right)=-\frac{\partial}{\partial h}\left[L_{d}\left(q_{0}, q_{1}, h\right)\right]
$$

where the time step has been inserted explicitly into the discrete Lagrangian in its third variable slot. One can motivate this definition of the energy using the variational principle and this definition may be viewed as a discrete form of the Hamilton-Jacobi equation. 
Example. For the discrete Lagrangian (2.2), the associated discrete energy is easily verified to be

$$
E_{d}^{\alpha}\left(q_{0}, q_{1}, h\right)=\frac{1}{2}\left(\frac{q_{1}-q_{0}}{h}\right)^{T} M\left(\frac{q_{1}-q_{0}}{h}\right)+V\left((1-\alpha) q_{0}+\alpha q_{1}\right) .
$$

In this example, we can write this as

$$
E_{d}^{\alpha}\left(q_{0}, q_{1}, h\right)=E\left((1-\alpha) q_{0}+\alpha q_{1},\left(\frac{q_{1}-q_{0}}{h}\right)\right)
$$

where $E(q, \dot{q})$ is the energy function associated with the original Lagrangian $L(q, \dot{q})$.

\subsection{Construction of Mechanical Integrators}

Assume we have a mechanical system defined by a Lagrangian $L: T Q \rightarrow \mathbb{R}$. If $Q$ is an arbitrary manifold, then one typically proceeds by embedding it within a vector space and treating $Q$ as a constraint manifold. We will assume for simplicity that $Q$ is a linear space.

We regard the discrete action sum (2.4) as an approximation to the action integral (2.5). Therefore, it is natural to choose the discrete Lagrangian $L_{d}$ : $Q \times Q \rightarrow \mathbb{R}$ to be an approximation:

$$
L_{d}\left(q_{0}, q_{1}\right) \approx \int_{0}^{h} L(q(t), \dot{q}(t)) d t
$$

where $q(t)$ is a true trajectory of the system that moves from $q_{0}$ at time $t=0$ to $q_{1}$ at time $t=h$. The right hand side of course is Jacobi's form of the exact solution $S\left(q_{0}, q_{1}, h\right)$ of the Hamilton-Jacobi equation.

We have already introduced one important class of discrete Lagrangians, namely $L_{d}^{\alpha}$ in equation (2.2). Another class of examples will be introduced below.

We introduce the useful evaluated acceleration notation:

$$
a_{k+\alpha}:=M^{-1}\left[-\nabla V\left((1-\alpha) q_{k}+\alpha q_{k+1}\right)\right] .
$$

With this notation, the algorithm (2.7) reads:

$$
\frac{1}{h^{2}}\left(q_{k+2}-2 q_{k+1}+q_{k}\right)=(1-\alpha) a_{k+1+\alpha}+\alpha a_{k+\alpha} .
$$

This is a second-order accurate implicit algorithm on $Q \times Q$. For a Lagrangian not of the special form above this need only be a first-order accurate algorithm for general $\alpha$, but is second order accurate for $\alpha=1 / 2$. 
A second choice of discrete Lagrangian is a symmetric version of the expression (2.2) for $L_{d}^{\alpha}$, defined by

$$
\begin{aligned}
L_{d}^{\mathrm{sym}, \alpha}\left(q_{0}, q_{1}\right)=\frac{h}{2} L\left((1-\alpha) q_{0}+\right. & \left.\alpha q_{1}, \frac{q_{1}-q_{0}}{h}\right) \\
& +\frac{h}{2} L\left(\alpha q_{0}+(1-\alpha) q_{1}, \frac{q_{1}-q_{0}}{h}\right),
\end{aligned}
$$

where, once again, $h \in \mathbb{R}^{+}$is the time step and $\alpha \in[0,1]$ is a real parameter.

For this discrete Lagrangian the corresponding discrete Euler-Lagrange equations also have a symmetric form. For $L$ given by (2.1) and using the evaluated acceleration notation (2.14), the DEL equations are:

$$
\begin{aligned}
& \frac{1}{h^{2}}\left(q_{k+2}-2 q_{k+1}+q_{k}\right) \\
& \quad=\frac{1}{2}(1-\alpha) a_{k+1+\alpha}+\frac{1}{2} \alpha a_{k+2-\alpha}+\frac{1}{2} \alpha a_{k+\alpha}+\frac{1}{2}(1-\alpha) a_{k+1-\alpha} .
\end{aligned}
$$

These equations define a second-order accurate, implicit algorithm for any parameter $\alpha$, and are still second-order accurate if we use an arbitrary Lagrangian $L$.

Both algorithms (2.15) and (2.17), derived from $L_{d}^{\alpha}$ and $L_{d}^{\text {sym, } \alpha}$ respectively, preserve the associated discrete symplectic form $\Omega_{L_{d}}$ and the discrete momentum map. By choosing $\alpha$ correctly these two algorithms recover many schemes known elsewhere under different names. Some examples of this are mentioned below.

\subsection{Representations of Variational Integrators}

One may think that the discrete symplectic form and momentum map that are conserved by the variational algorithm are somehow "concocted" to be conserved. This is not the case. Indeed, one can, via the discrete Legendre transform, transfer the algorithm to position-momentum space. Transferred to these variables, the algorithm will preserve the standard symplectic form $d q^{i} \wedge d p_{i}$ and the standard momentum map. If desired, one can then use the continuous Legendre transform to move the algorithm to $T Q$, where it will once again preserve the standard Lagrangian symplectic two-form $\Omega_{L}$ and the standard momenta.

To state these ideas more clearly, we summarize the three phase spaces we are using.

\begin{tabular}{c|c|c|c} 
Phase space & Local coords & Symp. form & Function \\
\hline$T^{*} Q$ & $(q, p)$ & $\Omega$ & Hamiltonian $H$ \\
$T Q$ & $(q, \dot{q})$ & $\Omega_{L}$ & Lagrangian $L$ \\
$Q \times Q$ & $\left(q_{0}, q_{1}\right)$ & $\Omega_{L_{d}}$ & Discrete Lagrangian $L_{d}$
\end{tabular}


The standard mappings between the phase spaces given above are:

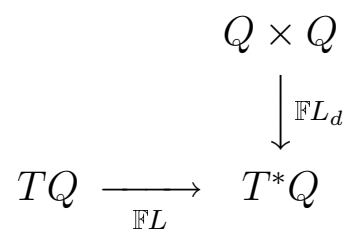

In this section we will be using the symmetric discrete Lagrangian $L_{d}^{\mathrm{sym}, \alpha}$ and assuming that the Lagrangian $L$ is given by (2.1). With these definitions the continuous and discrete fiber derivatives given by (2.9) and (2.8) have the form:

$$
\begin{aligned}
\mathbb{F} L(q, \dot{q}) & =(q, M \dot{q}) \\
\mathbb{F} L_{d}^{\mathrm{sym}, \alpha}\left(q_{0}, q_{1}\right) & =\left(q_{1}, M\left[\frac{1}{h}\left(q_{1}-q_{0}\right)+\frac{h}{2}\left(\alpha a_{0+\alpha}+(1-\alpha) a_{1-\alpha}\right)\right]\right) .
\end{aligned}
$$

The Variational Algorithm on $T^{*} Q$. Pushing the variational algorithm (2.17) forward with $\mathbb{F} L_{d}^{\text {sym }, \alpha}$ we obtain an implicit algorithm, which we denote

$$
\left(q_{k}, p_{k}\right) \mapsto\left(q_{k+1}, p_{k+1}\right),
$$

given by

$$
\begin{aligned}
& q_{k+1}=q_{k}+h M^{-1} p_{k}+\frac{h^{2}}{2}\left[(1-\alpha) a_{k+\alpha}+\alpha a_{k+1-\alpha}\right] \\
& p_{k+1}=p_{k}+h M\left(\frac{1}{2} a_{k+\alpha}+\frac{1}{2} a_{k+1-\alpha}\right) .
\end{aligned}
$$

This algorithm is second order accurate for any $\alpha$ and preserves the canonical symplectic form $\Omega$ and the standard momentum map.

Considering the particular case of $\alpha=1 / 2$ we see that the above algorithm can be written

$$
\frac{1}{h}\left(\begin{array}{c}
q_{k+1}-q_{k} \\
p_{k+1}-p_{k}
\end{array}\right)=\left(\begin{array}{c}
M^{-1}\left(\frac{p_{k+1}+p_{k}}{2}\right) \\
-\nabla V\left(\frac{q_{k+1}+q_{k}}{2}\right)
\end{array}\right)
$$

which, for $z=(q, p)$, has the more compact representation

$$
\frac{z_{k+1}-z_{k}}{h}=X_{H}\left(\frac{z_{k+1}+z_{k}}{2}\right) \text {. }
$$

where $X_{H}$ is the Hamiltonian vector field corresponding to the Hamiltonian

$$
H=\frac{1}{2} p^{T} M^{-1} p+V(q)
$$

This is the classical midpoint rule.

We summarize the results in the following: 
Theorem 2.1. The classical midpoint rule (2.18) is, via the discrete Legendre transformation, a variational algorithm with the choice $L_{d}=L_{d}^{\mathrm{sym}, \alpha}$ and $\alpha=$ $1 / 2$.

The variational algorithm on $T Q$. Next, we pull the variational algorithm back with $\mathbb{F} L$ from $T^{*} Q$ to $T Q$ to obtain an algorithm $\left(q_{k}, \dot{q}_{k}\right) \mapsto\left(q_{k+1}, \dot{q}_{k+1}\right)$ given by

$$
\begin{aligned}
& q_{k+1}=q_{k}+h \dot{q}_{k}+\frac{h^{2}}{2}\left[(1-\alpha) a_{k+\alpha}+\alpha a_{k+1-\alpha}\right] \\
& \dot{q}_{k+1}=\dot{q}_{k}+h\left(\frac{1}{2} a_{k+\alpha}+\frac{1}{2} a_{k+1-\alpha}\right) .
\end{aligned}
$$

This algorithm is second order accurate for any $\alpha$ and preserves the standard Lagrangian symplectic form $\Omega_{L}$ and the standard momentum map.

Aside from the midpoint rule mentioned above, a number of other classical integrators are also special cases of variational schemes. We get the shake algorithm with $L_{d}^{\alpha}$ for $\alpha=1$ (the Verlet algorithm is the unconstrained version of the shake algorithm). The Moser-Veselov discrete Lagrangian for the rigid body is constructed using $L_{d}^{\alpha}$ with either $\alpha=0$ or $\alpha=1$ (see Marsden, Pekarsky, and Shkoller [1999a,b] for details).

\section{The Newmark Algorithm is Variational}

The goal of this section is to prove that the Newmark scheme for conservative mechanical systems is variational, and to discuss some of the implications of this fact. The variational nature of the Newmark scheme and its performance is of particular interest because of its widespread use in finite element codes.

\subsection{Newmark Schemes}

We begin with a mechanical system on Euclidean $n$-space with a Lagrangian of the form

$$
L(q, \dot{q})=\frac{1}{2} \dot{q}^{T} M \dot{q}-V(q)
$$

where $q, \dot{q} \in \mathbb{R}^{n}, M$ is a constant symmetric and positive definite mass matrix, and $V$ is a given potential energy.

The corresponding Euler-Lagrange equations are, using vector notation,

$$
M \ddot{q}=-\nabla V(q)
$$


The Newmark family (Newmark [1959]) is one of the most widely used algorithms in structural dynamics. General references are Hughes [1987] and Geradin and Rixen [1988]. This family is usually written in the following way. Let $\gamma$ and $\beta$ be real numbers, usually taken between zero and one. Given $\left(q_{k}, \dot{q}_{k}\right)$, find $\left(q_{k+1}, \dot{q}_{k+1}\right)$ such that

$$
\begin{aligned}
& q_{k+1}=q_{k}+h \dot{q}_{k}+\frac{h^{2}}{2}\left[(1-2 \beta) a_{k}+2 \beta a_{k+1}\right] \\
& \dot{q}_{k+1}=\dot{q}_{k}+h\left[(1-\gamma) a_{k}+\gamma a_{k+1}\right],
\end{aligned}
$$

where we are using the convenient evaluated acceleration notation as earlier, defined by

$$
a_{k}=M^{-1}\left(-\nabla V\left(q_{k}\right)\right) .
$$

We recall that the Newmark algorithm is second order accurate if and only if $\gamma=1 / 2$, otherwise it is only consistent. Thus, one usually chooses $\gamma=1 / 2$. If $\beta=0$ then equation (3.3) is an explicit equation for $q_{k+1}$ in terms of $\left(q_{k}, \dot{q}_{k}\right)$, making the $\beta=0$ case known as explicit Newmark.

Simple comparison. The relationship between the Newmark algorithm and the variational schemes discussed earlier can be clearly seen by comparing the $\alpha=1 / 2, \beta=1 / 4$ Newmark:

$$
\begin{aligned}
& q_{k+1}=q_{k}+h \dot{q}_{k}+\frac{h^{2}}{2}\left(\frac{a_{k}+a_{k+1}}{2}\right) \\
& \dot{q}_{k+1}=\dot{q}_{k}+h\left(\frac{a_{k}+a_{k+1}}{2}\right)
\end{aligned}
$$

to the $L_{d}^{\text {sym, } \alpha}$ with $\alpha=1 / 2$ variational scheme:

$$
\begin{aligned}
& q_{k+1}=q_{k}+h \dot{q}_{k}+\frac{h^{2}}{2} a_{k+1 / 2} \\
& \dot{q}_{k+1}=\dot{q}_{k}+h a_{k+1 / 2} .
\end{aligned}
$$

Looking at these two equations, we see that Newmark averages forces, whereas the variational method evaluates forces at averaged positions. We now show that this is not, in fact, an essential difference, and the algorithms are in fact equivalent.

Newmark as an Update of Configuration Points. As we have already seen, it is possible to regard variational algorithms either as updates of positions $\left(q_{k}, q_{k+1}\right) \mapsto\left(q_{k+1}, q_{k+2}\right)$ or as updates of positions and velocities $\left(q_{k}, \dot{q}_{k}\right) \mapsto$ 
$\left(q_{k+1}, \dot{q}_{k+1}\right)$. This is also true of the Newmark algorithm, and to compare these two schemes it will be beneficial to work with both of them in position-update form.

Firstly note that the velocities in Newmark can be recovered from the positions using (3.3). This gives

$$
\begin{aligned}
\dot{q}_{k} & =\frac{1}{h}\left(q_{k+1}-q_{k}\right)-\frac{h}{2}\left[(1-2 \beta) a_{k}+2 \beta a_{k+1}\right] \\
\dot{q}_{k+1} & =\frac{1}{h}\left(q_{k+2}-q_{k+1}\right)-\frac{h}{2}\left[(1-2 \beta) a_{k+1}+2 \beta a_{k+2}\right] .
\end{aligned}
$$

Substituting these two expressions into the velocity update equation (3.4) and rearranging we obtain an explicit expression reminiscent of that for the variational algorithm:

$$
\begin{aligned}
& \frac{1}{h^{2}}\left(q_{k+2}-2 q_{k+1}+q_{k}\right) \\
& \quad-\beta a_{k+2}+(2 \beta-\gamma-1 / 2) a_{k+1}+(-\beta+\gamma-1 / 2) a_{k}=0 .
\end{aligned}
$$

Equivalence of Newmark and variational schemes. We will explore three ways in which the Newmark and variational schemes can be regarded as equivalent.

1. For certain parameter values, or for linear systems, the Newmark algorithm is the same as the transformation of the variational algorithm corresponding to $L_{d}^{\mathrm{sym}, \alpha}$ from $Q \times Q$ to $T Q$ using the Legendre transformations for $L_{d}^{\mathrm{sym}, \alpha}$ and $L$.

2. Under much more general assumptions, we will show that any Newmark trajectory with $\gamma=1 / 2$ and $\beta \leq 1 / 4$ is shadowed, in a sense we will make precise, by a trajectory of a variational algorithm.

3. Finally, we will establish that any Newmark algorithm with $\gamma=1 / 2$ is directly variational. That is, we will construct a discrete Lagrangian $L_{d}^{\beta}$ for which the discrete Euler-Lagrange equations are the Newmark method for $\gamma=1 / 2$ and the given $\beta$.

We will cover each type of equivalence in turn. The first way is more elementary, direct, and to some extent known. Of primary interest are the second and third methods, but we include the first for completeness and for motivation.

\subsection{Central Differences and Linear Systems}

We shall start the process of proving that Newmark is variational with the well known cases when $\beta=0$ and $\gamma=1 / 2$, or any Newmark scheme with $\gamma=1 / 2$ and $\beta \leq 1 / 4$ for a linear system. 
Theorem 3.1. The Newmark algorithm with $\beta=0$ and $\gamma=1 / 2$ is the same as the variational algorithm derived from $L_{d}^{\mathrm{sym}, \alpha}$ with $\alpha=0$ or $\alpha=1$ pushed forward to $T Q$ with the discrete Legendre transform.

Proof. Substituting the specified parameters into the Newmark equations (3.3) and (3.4), and into the variational equations on $T Q$ (2.19) we see that in both cases we obtain:

$$
\begin{aligned}
& q_{k+1}=q_{k}+h \dot{q}_{k}+\frac{h^{2}}{2} a_{k} \\
& \dot{q}_{k+1}=\dot{q}_{k}+h\left(\frac{1}{2} a_{k}+\frac{1}{2} a_{k+1}\right)
\end{aligned}
$$

and thus have equivalence.

Linear Systems. One can say a little more in the case of linear systems, that is, in the case of quadratic potentials. This is interesting because of the long history of Newmark in the linear finite element literature.

Theorem 3.2. For potentials $V$ so that $\nabla V$ is affine, Newmark with $\gamma=1 / 2$ and any $\beta \leq 1 / 4$ is the same as the $L_{d}^{\mathrm{sym}, \alpha}$ variational algorithm when $\alpha$ is chosen so that $\beta=\alpha(1-\alpha)$.

A special case of this is the variational nature of the (constant) average acceleration method $(\beta=1 / 4, \gamma=1 / 2)$ which is equivalent to the $L_{d}^{\mathrm{sym}, \alpha}$ $(\alpha=1 / 2)$ variational algorithm.

Proof. Once again this is a simple verification. For affine $\nabla V$ we have that $a_{k+\alpha}=(1-\alpha) a_{k}+\alpha a_{k+1}$ and substituting this into the variational equation (2.19) and rearranging we obtain

$$
\begin{aligned}
& q_{k+1}=q_{k}+h \dot{q}_{k}+\frac{h^{2}}{2}\left((1-2[\alpha(1-\alpha)]) a_{k}+2[\alpha(1-\alpha)] a_{k+1}\right) \\
& \dot{q}_{k+1}=\dot{q}_{k}+h\left(\frac{1}{2} a_{k}+\frac{1}{2} a_{k+1}\right),
\end{aligned}
$$

which is Newmark for $\beta=\alpha(1-\alpha)$ and $\gamma=1 / 2$. For any given $\beta \leq 1 / 4$ there is clearly an $\alpha$ so that $\beta=\alpha(1-\alpha)$.

\subsection{Shadowing of Newmark Trajectories}

We will now turn to a much more general class of Newmark algorithms and consider their action upon general nonlinear systems. In both this section and the next we are concerned with Newmark algorithms with the parameter $\gamma$ equal to $1 / 2$. 
This assumption is not as restrictive as it may initially appear. It is well known (see, for example, Hughes [1987]) that with $\gamma<1 / 2$ Newmark numerically dissipates energy and with $\gamma>1 / 2$ it numerically increases energy. For this reason one would not expect that Newmark with $\gamma \neq 1 / 2$ would be symplectic, and hence not variational.

The first way in which we establish the variational nature of Newmark is to show that any $\gamma=1 / 2, \beta \leq 1 / 4$ Newmark trajectory will be shadowed by a variational trajectory, and vice versa. By this, we mean that there is some parameter $\alpha$ so that each point $x_{k}$ of the Newmark trajectory is equal to the interpolation $(1-\alpha) q_{k}+\alpha q_{k+1}$ of two points $q_{k}, q_{k+1}$ of the variational trajectory. This can be clearly seen in Figure 3.1, which shows a Newmark and a variational trajectory shadowing each other.

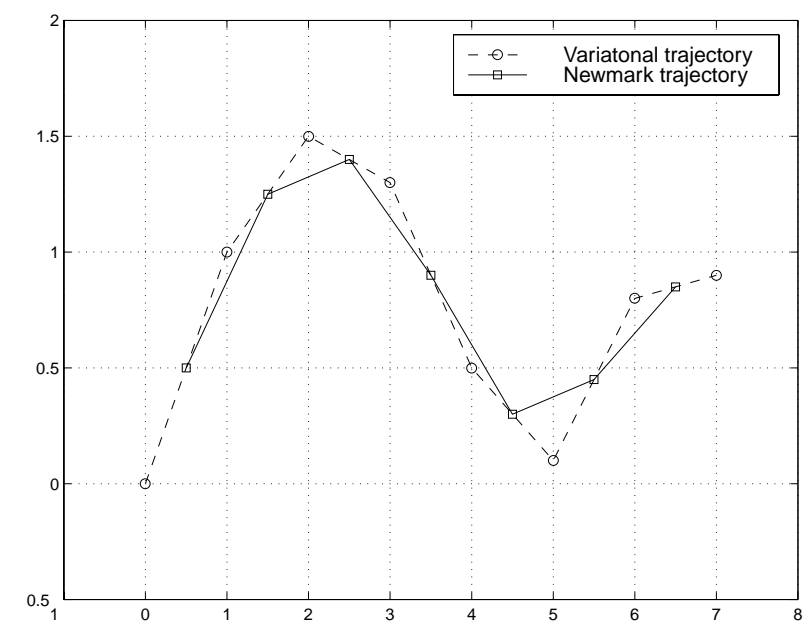

Figure 3.1: A Newmark trajectory (solid line) and its "shadow" variational trajectory (dashed line).

In this section we will always use $x_{k}$ to denote points on a Newmark trajectory and $q_{k}$ to denote points on a variational trajectory. In this equivalence we are only interested in the trajectories in configuration space $Q$ and do not consider the velocities. The reason this is possible is that Newmark can be regarded as a position-update only algorithm, as discussed in Section 3.1.

We now give a lemma containing the fundamental fact behind the shadowing variational nature of Newmark.

Lemma 3.3. For any $q_{0}, q_{1}, q_{2}$ and $q_{3}$ lying on a trajectory of the $L_{d}^{\alpha}$ variational algorithm for some $\alpha$, the points $x_{0}, x_{1}$ and $x_{2}$ formed by

$$
x_{k}=(1-\alpha) q_{k}+\alpha q_{k+1}
$$

satisfy the position-update Newmark algorithm with $\beta=\alpha(1-\alpha)$ and $\gamma=1 / 2$. 
Proof. Begin by noting that, by assumption, the two triples $\left(q_{0}, q_{1}, q_{2}\right)$ and $\left(q_{1}, q_{2}, q_{3}\right)$ both satisfy the discrete Euler-Lagrange equations (2.15).

Now substitute the expressions for $x_{k}$ and $\beta=\alpha(1-\alpha)$ into the left-hand side of the position-update Newmark algorithm (3.6) to obtain

$$
\begin{aligned}
& \frac{M}{h^{2}}\left(-\alpha q_{3}+(3 \alpha-1) q_{2}+(2-3 \alpha) q_{1}+(\alpha-1) q_{0}\right) \\
& \quad-\alpha(1-\alpha) D V\left((1-\alpha) q_{2}+\alpha q_{3}\right) \\
& \quad-\left(2 \alpha^{2}-2 \alpha+1\right) D V\left((1-\alpha) q_{1}+\alpha q_{2}\right) \\
& \quad-\alpha(1-\alpha) D V\left((1-\alpha) q_{0}+\alpha q_{1}\right) .
\end{aligned}
$$

This expression can be rearranged to give

$$
\begin{aligned}
=\alpha & {\left[\frac{M}{h^{2}}\left(-q_{3}+2 q_{2}-q_{1}\right)-(1-\alpha) D V\left((1-\alpha) q_{2}+\alpha q_{3}\right)\right.} \\
& \left.-\alpha D V\left((1-\alpha) q_{1}+\alpha q_{2}\right)\right] \\
+ & (1-\alpha)\left[\frac{M}{h^{2}}\left(-q_{2}+2 q_{1}-q_{0}\right)-(1-\alpha) D V\left((1-\alpha) q_{1}+\alpha q_{2}\right)\right. \\
& \left.-\alpha D V\left((1-\alpha) q_{0}+\alpha q_{1}\right)\right] .
\end{aligned}
$$

Each of the bracketed expressions is just the discrete Euler-Lagrange equations satisfied by the sets $q_{1}, q_{2}, q_{3}$ and $q_{0}, q_{1}, q_{2}$, and thus the entire expression is zero, as claimed.

Theorem 3.4. Taking $\gamma=1 / 2$, given any parameter $\beta$ with $0 \leq \beta \leq 1 / 4$ and any initial conditions $\left(x_{0}, \dot{x}_{0}\right)$ for Newmark, there exists, for small time steps $h$, initial conditions $\left(q_{0}, q_{1}\right)$ and parameter $\alpha$ for the $L_{d}^{\alpha}$ variational algorithm so that the Newmark and variational trajectories are related by $x_{k}=(1-\alpha) q_{k}+$ $\alpha q_{k+1}$ for all $k$. That is, the trajectories shadow each other for all time.

Conversely, given a parameter $\alpha$ and initial conditions $\left(q_{0}, q_{1}\right)$ for the $L_{d}^{\alpha}$ variational algorithm, there exists initial conditions $\left(x_{0}, \dot{x}_{0}\right)$ and parameter $\beta$ so that the same conclusion holds.

Proof. The proof of this result is essentially a repeated application of the preceding lemma as time increases. The parameters $\alpha$ and $\beta$ must always be taken so that $\beta=\alpha(1-\alpha)$. Note that this implies two possible values of $\alpha$ for any given $\beta \leq 1 / 4$, except for the "midpoint" case $\beta=1 / 4$ and $\alpha=1 / 2$.

Given initial conditions $\left(x_{0}, \dot{x}_{0}\right)$ for Newmark, compute $\left(x_{1}, \dot{x}_{1}\right)$ with one 
step of Newmark. Now we must find $q_{0}, q_{1}$ and $q_{2}$ so that

$$
\begin{aligned}
D_{1} L_{d}^{\alpha}\left(q_{1}, q_{2}\right)+D_{2} L_{d}^{\alpha}\left(q_{0}, q_{1}\right) & =0 \\
(1-\alpha) q_{0}+\alpha q_{1} & =x_{0} \\
(1-\alpha) q_{1}+\alpha q_{2} & =x_{1} .
\end{aligned}
$$

This is done by the implicit function theorem. First note that if we multiply the first equation by $h$ so that it is regular at $h=0$, then for $h=0$, we have the trivial solution $x_{0}=x_{1}=q_{0}=q_{1}=q_{2}$. Now we linearize around this solution; the relevant Jacobian determinant is computed at this solution to be $\left(1+\alpha^{2}\right)$ det $M$, which is not zero, so we have solvability for small $h$.

For the converse, given $\left(q_{0}, q_{1}\right)$, step twice with the variational algorithm to find $q_{2}$ and $q_{3}$, form $x_{0}$ and $x_{1}$ by interpolation and then use the position equation (3.3) of Newmark to find $v_{0}$. That is,

$$
\dot{q}_{0}=\frac{1}{h}\left(q_{1}-q_{0}\right)-\frac{h}{2}\left[(1-2 \beta) a_{0}+2 \beta a_{1}\right] .
$$

This completes the proof of the shadowing variational nature of the Newmark scheme.

It may appear at first that the properties of the variational algorithm will not be inherited by the Newmark method, as we have only established equivalence of the position-update forms of the algorithms in a shadowing sense. In fact, we can regard the 'shadowing' of trajectories as an implicitly defined nonlinear coordinate change, and the transformation $\left(q_{k}, q_{k+1}\right) \mapsto\left(q_{k}, \dot{q}_{k}\right)$ for both Newmark and the variational method is also simply a coordinate change. Thus we have that the Newmark and variational algorithms are simply the coordinate transformed versions of each other. The advantage in the shadowing result is that it provides clear physical insight into the relationship between the simple $L_{d}^{\alpha}$ variational algorithm and Newmark.

\subsection{Newmark Itself is Variational}

Now we are ready to show the sense in which Newmark itself is directly variational, and thus symplectic and momentum conserving, for $\gamma=1 / 2$.

This technique was suggested by Y.B. Suris, who made a key remark to us after viewing an early version of this work. We are most grateful to him for the elegant method of constructing a discrete Lagrangian which generates the Newmark algorithm.

To construct a discrete Lagrangian for $\gamma=1 / 2$ Newmark, we begin by noting that for $\gamma=1 / 2$ the position-update form of Newmark (3.6) can be 
written

$$
\begin{aligned}
\frac{1}{h}\left[\left(q_{k+2}-\beta h^{2} M^{-1}\right.\right. & \left.\nabla V\left(q_{k+2}\right)\right)-2\left(q_{k+1}-\beta h^{2} M^{-1} \nabla V\left(q_{k+1}\right)\right) \\
& \left.+\left(q_{k}-\beta h^{2} M^{-1} \nabla V\left(q_{k}\right)\right)\right]+h M^{-1} \nabla V\left(q_{k+1}\right)=0 .
\end{aligned}
$$

Making the coordinate change $q_{k} \mapsto x_{k}=\eta^{\beta}\left(q_{k}\right)$ defined by

$$
x_{k}=\eta^{\beta}\left(q_{k}\right)=q_{k}-\beta h^{2} M^{-1} \nabla V\left(q_{k}\right)
$$

and introducing a modified potential $\tilde{V}\left(x_{k}\right)$, we can write Newmark as

$$
\frac{1}{h}\left(x_{k+2}-2 x_{k+1}+x_{k}\right)+h M^{-1} \nabla \tilde{V}\left(x_{k}\right)=0,
$$

where we require that $\tilde{V}\left(x_{k}\right)$ is related to $V\left(q_{k}\right)$ by

$$
\nabla \tilde{V}\left(x_{k}\right)=\nabla V\left(q_{k}\right)
$$

for $x_{k}=\eta^{\beta}\left(q_{k}\right)$. Equation (3.8), however, is simply the discrete Euler-Lagrange equations for the discrete Lagrangian

$$
L_{d}\left(x_{0}, x_{1}\right)=h \frac{1}{2}\left(\frac{x_{1}-x_{0}}{h}\right)^{T} M\left(\frac{x_{1}-x_{0}}{h}\right)-h \tilde{V}\left(x_{0}\right) .
$$

We now summarize this as a theorem, and prove that the modified potential $\tilde{V}$ actually exists.

Theorem 3.5. The Newmark method with $\gamma=1 / 2$ and any $\beta$ is the discrete Euler-Lagrange equations for the discrete Lagrangian $L_{d}^{\beta}$ defined by

$$
L_{d}^{\beta}\left(q_{0}, q_{1}\right)=h \frac{1}{2}\left(\frac{\eta^{\beta}\left(q_{1}\right)-\eta^{\beta}\left(q_{0}\right)}{h}\right)^{T} M\left(\frac{\eta^{\beta}\left(q_{1}\right)-\eta^{\beta}\left(q_{0}\right)}{h}\right)-h \tilde{V}\left(\eta^{\beta}\left(q_{0}\right)\right)
$$

where $\eta^{\beta}\left(q_{k}\right)=q_{k}-h^{2} \beta M^{-1} \nabla V\left(q_{k}\right)$ and $\tilde{V}$ is defined so that $\nabla \tilde{V}\left(\eta^{\beta}\left(q_{k}\right)\right)=$ $\nabla V\left(q_{k}\right)$ for all $q_{k}$.

Proof. If such a discrete Lagrangian is well-defined then the above calculations show that the discrete Euler-Lagrange equations will give the Newmark algorithm with $\gamma=1 / 2$ and the given $\beta$. We need only check that the modified potential function $\tilde{V}$ exists, given that it must satisfy the relation

$$
\nabla \tilde{V}\left(\eta^{\beta}\left(q_{k}\right)\right)=\nabla V\left(q_{k}\right)
$$


We introduce the following notation:

$$
\begin{aligned}
& \eta^{\beta}(q)=q-\beta h^{2} M^{-1} \nabla V(q) \\
& \xi^{\beta}(x)=\left(\eta^{\beta}\right)^{-1}(x) \\
& X(x)=\nabla V\left(\xi^{\beta}(x)\right),
\end{aligned}
$$

and observe that we are trying to establish that $X(x)$ is the gradient of some function. A necessary and sufficient condition for this is for the following to hold:

$$
\frac{\partial X_{i}}{\partial x^{j}}-\frac{\partial X_{j}}{\partial x^{i}}=0 \quad \text { for all } i, j
$$

Using $\nabla^{2} V$ to denote the matrix of second partial derivatives of $V$ and $D \xi^{\beta}$ to denote the matrix of derivatives of $\xi^{\beta}$ we see that the left hand side of (3.11) is

$$
\left(\nabla^{2} V D \xi^{\beta}\right)^{T}-\left(\nabla^{2} V D \xi^{\beta}\right) .
$$

Computing $D \eta^{\beta}=I-\beta h^{2} M^{-1} \nabla^{2} V$ it is clear that the symmetry of $M$ and $\nabla^{2} V$ implies that $D \eta^{\beta}$ satisfies the relation $\left(D \eta^{\beta}\right)^{T} \nabla^{2} V=\nabla^{2} V D \eta^{\beta}$. Using the fact that $D \eta^{\beta}$ and $D \xi^{\beta}$ are inverses allows this to be rearranged to yield $\nabla^{2} V D \xi^{\beta}=\left(D \xi^{\beta}\right)^{T} \nabla^{2} V$, and using again the symmetry of $\nabla^{2} V$ shows that (3.12) is zero and thus condition (3.11) is satisfied.

Since we are working in $\mathbb{R}^{n}$, we conclude that a function $\tilde{V}$ with the desired

property (3.10) exists, and so $L_{d}^{\beta}$ is well-defined and generates the Newmark method for $\gamma=1 / 2$ and any $\beta$.

\subsection{Consequences of Newmark's Variational Nature}

There are three ways of interpreting the variational and symplectic nature of the Newmark integrator. Firstly, one can consider the Newmark and variational schemes to be essentially the same, except the variational method has the right form to exactly conserve the momenta. As the two methods have almost identical implementations, one could simply change to using the variational integrator.

A second way to interpret this result is to realize that it implies that Newmark will exactly preserve momenta and a symplectic form, except they will not be quite the obvious ones we are used to writing down. It is possible to derive expressions for these momenta and the non-canonical symplectic form conserved by Newmark, but the expressions can be rather unwieldy. We give an example of this below. The observation of Simo, Tarnow and Wong [1992] that central differences Newmark preserves momenta evaluated at midpoints seems to be consistent with this. 
Example. Let us work out the nature of the Newmark conserved quantity associated to the first coordinate being cyclic. We considered this example in the introduction for the variational algorithm. As usual, we consider a Lagrangian of the usual form of kinetic minus potential energy:

$$
L(q, \dot{q})=\frac{1}{2} \dot{q}^{T} M \dot{q}-V(q) .
$$

Assume that $V$ is independent of the first component $q^{1}$ of $q$, so that $p_{1}=[M \dot{q}]_{1}$ is a constant of the true continuous motion.

If we use the Newmark method to simulate this system, obtaining a trajectory $\left\{q_{k}\right\}$, then we know that the corresponding discrete momentum map derived from $L_{d}^{\beta}$ will be conserved. Computing this gives

$$
J_{d}=\left[M\left(\frac{\eta^{\beta}\left(q_{k}\right)-\eta^{\beta}\left(q_{k-1}\right)}{h}\right) D \eta^{\beta}\left(q_{k}\right)\right]_{1}
$$

which will be a constant, that is, independent of $k$.

Note that $\eta^{\beta}$ is the identity plus a term of order $h^{2}$. The naive approximation of $p_{1}$, using a simple finite difference approximation of $\dot{q}$, is thus related to the true discrete momentum by

$$
J_{d}=\left[M\left(\frac{q_{k}-q_{k-1}}{h}\right)\right]_{1}+\mathcal{O}\left(h^{2}\right)
$$

showing that the usual discretization of momentum will be preserved up to order $h^{2}$.

More useful than actually finding the exactly conserved structures is to use them in a third interpretation of the variational nature of Newmark. The existence of nearby conserved quantities allows us to conclude the following.

Theorem 3.6. For sufficiently small time-step $h$, a regular value of the momentum, and assuming that the solutions computed by the Newmark algorithm (3.3), (3.4) with $\gamma=1 / 2$ are bounded for all time, then these solutions will have bounded momentum error, uniformly for all time, and this error will tend to zero as $h$ tends to zero.

Proof. This follows from the fact that the corresponding exactly conserved quantity for the variational integrator will be evaluated at the interpolated shadowing points for the Newmark algorithm and that the algorithmic momentum level sets are uniformly close (in bounded regions) to the momentum level sets for $h$ small.

Using the correspondence between the Newmark and variational schemes, which are symplectic, we can also use results applicable to symplectic integrators to understand the behavior of Newmark. An example of this is the work on the energy behavior of symplectic integrators (see, for example, Sanz-Serna and Calvo [1994] and Hairer and Lubich [1997] and references therein). 


\subsection{Minimization Structure of the Newmark Algorithm}

In this section we consider a different way of writing the Newmark algorithm using optimization methods. This will be particularly useful when we come to algorithms for dissipative and forced systems, so we include forces in the formulation already here.

We consider a set of equations of motion of the form:

$$
M \ddot{q}+f^{\text {int }}(q, \dot{q})=f^{\text {ext }}(t)
$$

with $q(t) \in \mathbb{R}^{n}$, and where we regard $f^{\text {ext }}(t)$ as a given external force. As for the internal force, we postulate the existence of a conservative potential $V(q)$ and a dissipative potential $\varphi(q, \dot{q})$ such that:

$$
f^{\text {int }}(q, \dot{q})=\frac{\partial V(q)}{\partial q}+\frac{\partial \varphi(q, \dot{q})}{\partial \dot{q}}
$$

Dissipative potentials of this sort can be regarded as Rayleigh dissipation functions; we will discuss these in greater detail in the second half of this paper.

We discretize the equations of motion using the Newmark scheme as follows:

$$
\begin{aligned}
q_{k+1} & =q_{k}+h \dot{q}_{k}+\frac{h^{2}}{2}\left[(1-2 \beta) a_{k}+2 \beta a_{k+1}\right] \\
M a_{k+1}+f_{k+1}^{\text {int }} & =f_{k+1}^{\text {ext }} \\
\dot{q}_{k+1} & =\dot{q}_{k}+h\left[(1-\gamma) a_{k}+\gamma a_{k+1}\right] .
\end{aligned}
$$

We define

$$
q_{k+1}^{\text {pre }}=q_{k}+h \dot{q}_{k}+\frac{h^{2}}{2}\left[(1-2 \beta) a_{k}\right]
$$

so that (3.15) reads

$$
q_{k+1}=q_{k+1}^{\mathrm{pre}}+\beta h^{2} a_{k+1}
$$

To close this set of equations, we need to supply a relation between $f_{k+1}^{\text {int }}$ and $q_{k+1}$. To this end, following Radovitzky and Ortiz [1999], and Ortiz and Stainier [1999], we introduce the effective incremental potential

$$
V_{k}\left(q_{k+1}\right)=V\left(q_{k+1}\right)+h \varphi\left(q_{k+\sigma}, \frac{q_{k+1}-q_{k}}{h}\right),
$$

where

$$
q_{k+\sigma}=(1-\sigma) q_{k}+\sigma q_{k+1}
$$


and $\sigma \in[0,1]$, and write:

$$
f_{k+1}^{\mathrm{int}}=\frac{\partial V_{k}\left(q_{k+1}\right)}{\partial q_{k+1}}
$$

which is consistent with (3.14) as $h \rightarrow 0$ for any choice of $\sigma$.

The above algorithm can be recast in optimization form as follows. Combine (3.15) and (3.16) to get:

$$
M \frac{q_{k+1}-q_{k+1}^{\mathrm{pre}}}{h^{2}}+\beta f_{k+1}^{\mathrm{int}}=\beta f_{k+1}^{\mathrm{ext}} .
$$

Clearly, this is the Euler-Lagrange equation of the function:

$$
f\left(q_{k+1}\right)=\frac{1}{2 h^{2}}\left(q_{k+1}-q_{k+1}^{\mathrm{pre}}\right)^{T} M\left(q_{k+1}-q_{k+1}^{\mathrm{pre}}\right)+\beta V_{k}\left(q_{k+1}\right)-\beta f_{k+1}^{\mathrm{ext}} \cdot q_{k+1} .
$$

Therefore, under appropriate convexity conditions on $V$ and $\varphi$, the updated configuration follows as the solution of the minimum principle:

$$
\min _{q_{k+1}} f\left(q_{k+1}\right)
$$

Once $q_{k+1}$ is determined, the internal forces are computed from eq. (3.20) and subsequently the velocities are updated using (3.17).

Some remarks about these results are in order:

1. The minimum principle for non-convex potentials can be replaced by a principle of stationarity. However, cases in which one has a minimum are useful to stress for they can be useful for error estimates and also for the use of optimization techniques, as in Ortiz and Stainier [1999], Kane, Repetto, Ortiz and Marsden [1999] and Kane, Marsden, Ortiz and Pandolfi [1999].

2. The minimization principle operates on the non-explicit part of the Newmark algorithm. Thus, in this view, it can be regarded as a two step procedure; first one computes the predictor point by an explicit formula and then, secondly, one corrects this with the minimization principle. This use of the term "predictor" is special to this formulation. We shall use the term slightly differently in later sections.

3. Notice that in the case of conservative systems (so there are no external forces or dissipative potentials) this scheme is literally the Newmark scheme for conservative systems, which, as we have shown, is equivalent to a variational scheme. Thus, the same scheme has both a variational (and hence symplectic) interpretation as well as obeying an interesting minimization principle. These facts together should be useful in extending the analytical results on Newmark beyond what we have done. 


\section{Numerical Tests for Conservative Systems}

\subsection{Example System}

To illustrate the performance of some of the algorithms discussed in the preceding sections we consider a simple conservative system. The example chosen here is a two degree of freedom non-linear oscillator, consisting of a particle with unit mass moving in the plane with trajectory $q(t) \in \mathbb{R}^{2}$ under the influence of the potential

$$
V(q)=\|q\|^{2}\left(\|q\|^{2}-1\right)^{2} .
$$

The Lagrangian describing this problem is thus

$$
L(q, \dot{q})=\frac{1}{2} \dot{q}^{T} \dot{q}-V(q)
$$

and the equations of motion are the corresponding Euler-Lagrange equations.

Trajectories of this example system have two conserved quantities. First, the mechanical nature of the system implies that energy is conserved. Second, the fact that the potential $V$, and hence the Lagrangian $L$, is radially symmetric implies conservation of angular momentum.

We are interested here in the extent to which the different integration schemes actually preserve these two quantities. This is an issue related to, but different from, the absolute accuracy of trajectories. Although symplectic integration schemes frequently exhibit improved trajectory accuracy, this is not guaranteed. Nonetheless, we focus here on the preservation of invariants of the system since these properties will be reflected in an interesting way for the Newmark scheme.

\subsection{Tested Algorithms}

The algorithms for which we present results here are:

- Implicit Newmark: Newmark with $\beta=1 / 4, \gamma=1 / 2$

- Explicit Newmark: Newmark with $\beta=0, \gamma=1 / 2$

- Variational: $L_{d}^{\mathrm{sym}, \alpha}$ variational integrator with $\alpha=1 / 2$

- Runge-Kutta: 4th order, fixed time-step

- Benchmark: MATLAB 5.3 ODE113 (Predictor-corrector)

The 4th order Runge-Kutta method is a classical integrator which makes no use of the mechanical nature of the system. It is included to demonstrate the behavior which occurs if one treats the system as an arbitrary set of equations, rather than taking advantage of their mechanical structure. 
All the integrators except for the benchmark code are run with the same step size of $h=0.2$ to provide a reasonable comparison between them. The benchmark code is a high order, multi-step, predictor-corrector method which we run with a very small step size. On an example this simple, the benchmark method can be regarded as essentially identical to the true solution.

The energy at each time-step is evaluated as the energy on $T Q$. That is, for the variational method we use the pull-back of the true energy under the discrete fiber derivative.

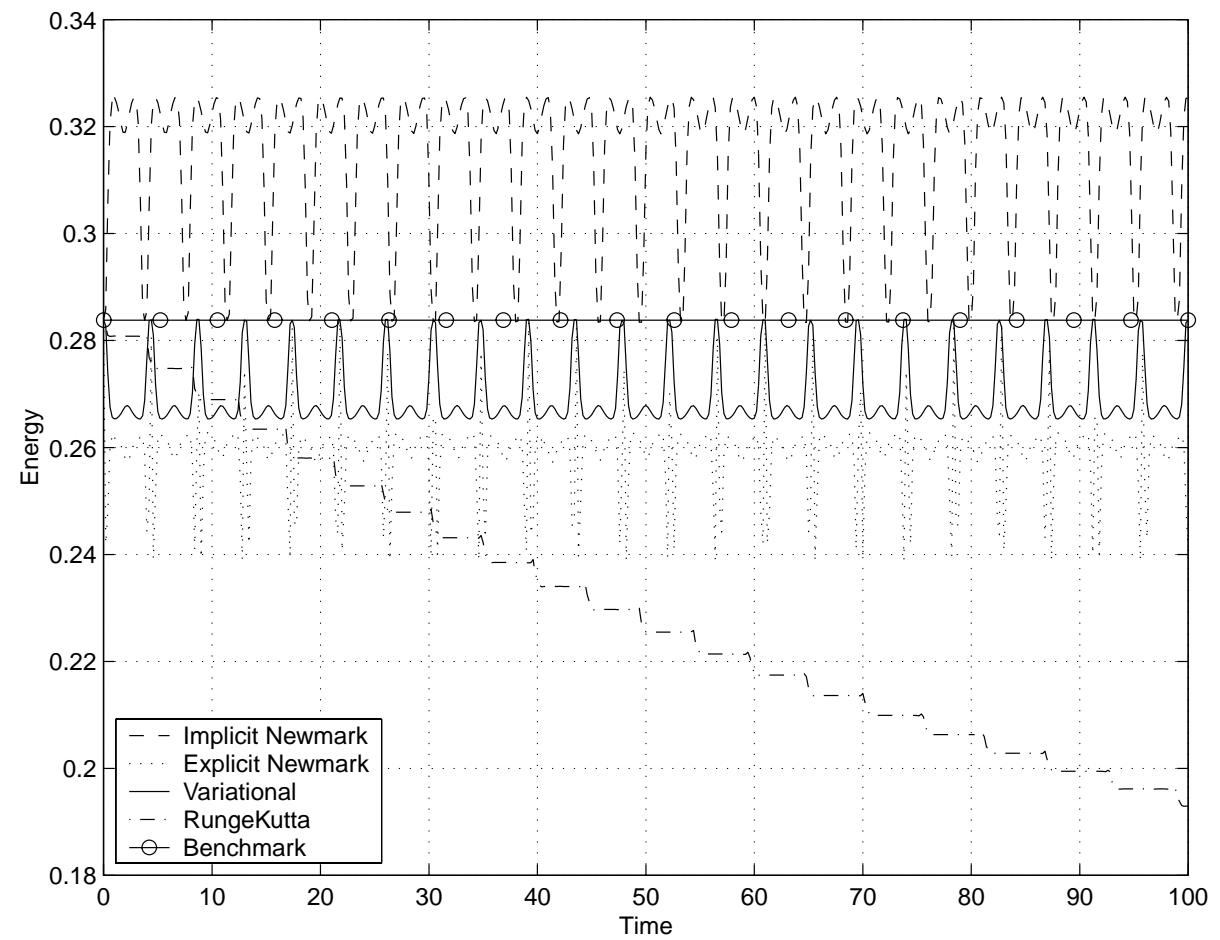

Figure 4.1: Energy behavior of integrators for a conservative system. Note the long-time stable behavior typical of the variational method, as contrasted to classical methods such as Runge-Kutta. The stable behavior of Newmark is explained by its variational nature.

\subsection{Results}

The energy behavior of the various integrators is shown for a short time in Figure 4.1. The same pattern is observed if the simulation is carried out for essentially arbitrarily long times. It is immediately apparent from this figure that the Newmark and variational methods have qualitatively different behavior to the Runge-Kutta technique. This fluctuating energy behavior of variational schemes is typical of symplectic methods, and provides a clear indication that Newmark is in fact symplectic. 


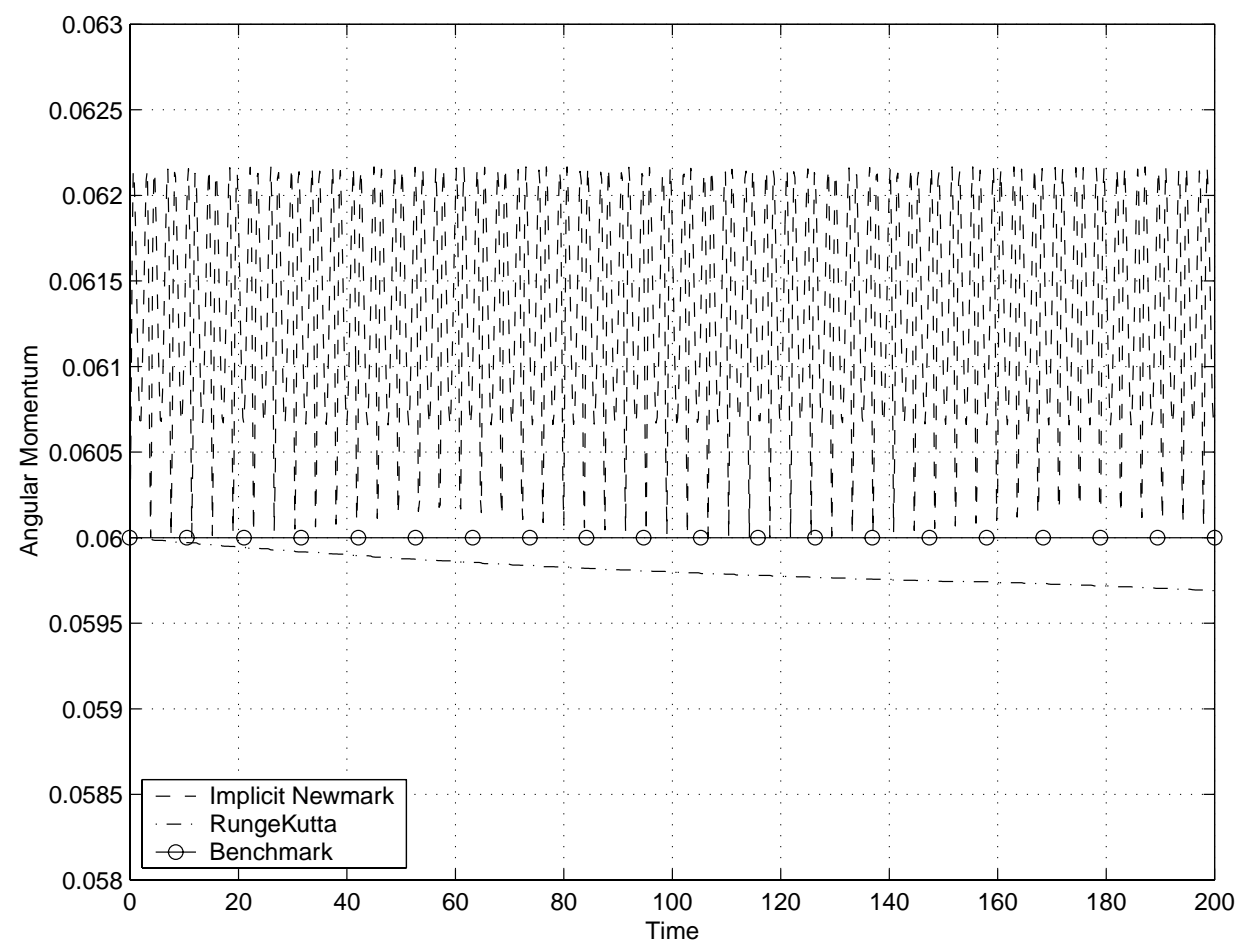

Figure 4.2: Momentum behavior of integrators for a conservative system. Variational results are not plotted as they exactly conserve momenta. Note the predicted long-time stable behavior of Newmark, as contrasted against the divergent behavior of methods such as Runge-Kutta.

The evolution of the angular momentum with the various integrators is plotted in Figure 4.2. The results for the variational algorithm and the explicit Newmark method are not shown, because they exactly conserve the angular momentum of the system, as explained previously. In this figure we see clearly the Newmark behavior implied by Theorem 3.6 and that result shows that this oscillatory behavior will persist indefinitely. While a standard integrator will have divergent momentum behavior, the fact that Newmark is variational under a near-identity change of coordinates forces the angular momentum to be almost conserved, with at most the finite fluctuations seen here.

We must caution against attempts to interpret the variations in energy as percentage or relative errors. This is because the energy of the system is only meaningful up to an additive constant and so the initial energy, or energy at any given time, is entirely arbitrary. It is also not significant that the energies of the various trajectories all lie either above or below the exact value. This is due to the simple nature of the example system, and is not apparent for more complex problems.

Note that the accuracy of the benchmark algorithm is demonstrated by 
the fact that it preserves the energy and momentum to within almost machine precision. This will be of use when we perform numerical tests on dissipative systems for which the true behavior cannot be analytically calculated.

\section{Numerical Algorithms for Systems with Fric- tion}

Now we consider nonconservative systems; those with forcing and in particular, those with dissipation. The dissipation considered here is of simple Rayleigh dissipation type. In other publications (Kane, Marsden, Ortiz and Pandolfi [1999]) we use these methods in collision problems with Coulomb friction.

We consider a given Lagrangian $L(q, \dot{q})$ and associated dissipative systems with Rayleigh type dissipation of the following form:

$$
\frac{d}{d t} \frac{\partial L}{\partial \dot{q}^{i}}-\frac{\partial L}{\partial q^{i}}=-\frac{\partial \varphi}{\partial \dot{q}}(q, \dot{q})
$$

where $\varphi(q, \dot{q})$ is a given dissipative potential. We are specifically interested in the case when $L$ has the form

$$
L(q, \dot{q})=\frac{1}{2} \dot{q}^{T} M \dot{q}-V(q),
$$

where $q \in \mathbb{R}^{n}, M$ is a constant mass matrix (a positive definite symmetric $n \times n$ matrix) and $V$ is a given potential energy function, so that the equations take the form

$$
M \ddot{q}=-\nabla V(q)-\frac{\partial \varphi}{\partial \dot{q}}(q, \dot{q}) .
$$

Let the energy be denoted

$$
E(q, \dot{q})=\frac{1}{2} \dot{q}^{T} M \dot{q}+V(q)
$$

and recall the usual energy equation

$$
\frac{d}{d t} E=-\left\langle\dot{q}, \frac{\partial \varphi}{\partial \dot{q}}(q, \dot{q})\right\rangle .
$$

The standard example is of course the case

$$
\varphi(q, \dot{q})=\frac{1}{2} \dot{q}^{T} R \dot{q}
$$

where $R$ is a positive definite symmetric matrix, so that the energy equation becomes

$$
\frac{d}{d t} E=-\dot{q}^{T} R \dot{q} \leq 0
$$


Our aim is to develop algorithms that have good energy decay properties in the sense that the algorithms predict the correct energy drop after a long integration run. We shall consider three types of algorithms (not necessarily in order of performance or preference):

1. Algorithms based on a discretization of the Lagrange d'Alembert principle as a generalization of variational integrators

2. Two step integration methods that separate the algorithms into conservative and dissipative parts

3. Minimization algorithms that are directly related to the Newmark algorithm with forces. Here the steps are based on explicit-implicit splits.

We will consider these in turn.

\subsection{Discrete Lagrange-d'Alembert Principle}

Recall that the (continuous) integral Lagrange-d'Alembert principle is

$$
\delta \int L(q(t), \dot{q}(t)) d t+\int F(q(t), \dot{q}(t)) \cdot \delta q d t=0 .
$$

We define the discrete Lagrange-d'Alembert principle to be

$$
\delta \sum L_{d}\left(q_{k}, q_{k+1}\right)+\sum\left[F_{d}^{-}\left(q_{k}, q_{k+1}\right) \cdot \delta q_{k}+F_{d}^{+}\left(q_{k}, q_{k+1}\right) \cdot \delta q_{k+1}\right]=0,
$$

where $L_{d}$ is the discrete Lagrangian and $F_{d}^{-}$and $F_{d}^{+}$are the left and right discrete forces.

The equation (5.2) defines an integrator $\left(q_{k}, q_{k+1}\right) \mapsto\left(q_{k+1}, q_{k+2}\right)$ given implicitly by the forced discrete Euler-Lagrange equations:

$$
D_{1} L_{d}\left(q_{k+1}, q_{k+2}\right)+D_{2} L_{d}\left(q_{k}, q_{k+1}\right)+F_{d}^{-}\left(q_{k+1}, q_{k+2}\right)+F_{d}^{+}\left(q_{k}, q_{k+1}\right)=0 .
$$

Example. The discrete force analogues of the symmetric discrete Lagrangian $L_{d}^{\mathrm{sym}, \alpha}$ given in equation (2.16) are:

$$
\begin{aligned}
F_{d}^{\mathrm{sym}, \alpha-}\left(q_{0}, q_{1}\right) \cdot \delta q_{0}=h \frac{1}{2}[(1 & -\alpha) F\left(q_{0+\alpha}, \frac{q_{1}-q_{0}}{h}\right) \\
& \left.+\alpha F\left(q_{1-\alpha}, \frac{q_{1}-q_{0}}{h}\right)\right] \cdot \delta q_{0} \\
F_{d}^{\mathrm{sym}, \alpha+}\left(q_{0}, q_{1}\right) \cdot \delta q_{1}=h \frac{1}{2}[\alpha F & \left(q_{0+\alpha}, \frac{q_{1}-q_{0}}{h}\right) \\
& \left.+(1-\alpha) F\left(q_{1-\alpha}, \frac{q_{1}-q_{0}}{h}\right)\right] \cdot \delta q_{1}
\end{aligned}
$$


where the interpolated positions are $q_{k+\alpha}=(1-\alpha) q_{k}+\alpha q_{k+1}$.

With these discrete forces and the discrete Lagrangian given by $L_{d}^{\mathrm{sym}, \alpha}$, the forced discrete Euler-Lagrange equations (5.3) define a second order accurate integrator for arbitrary Lagrangian $L$ and arbitrary force $F$. In the particular case of $L=(1 / 2) \dot{q}^{T} M \dot{q}-V(q)$ and $F=F(\dot{q})$ the integrator has the special form:

$$
\begin{aligned}
\frac{1}{h}\left(-q_{k+2}+2 q_{k+1}-q_{k}\right)+ & \frac{h}{2}(1-\alpha) a_{k+1+\alpha}+\frac{h}{2} \alpha a_{k+2-\alpha}+\frac{h}{2} \alpha a_{k+\alpha}+\frac{h}{2}(1-\alpha) a_{k+1-\alpha} \\
& +\frac{h}{2} F\left(\frac{q_{k+2}-q_{k+1}}{h}\right)+\frac{h}{2} F\left(\frac{q_{k+1}-q_{k}}{h}\right)=0
\end{aligned}
$$

Some numerics and tests of this variational Lagrange-d'Alembert method are given below.

\subsection{The Newmark Algorithm with Forcing is Variational}

It is interesting to note that for forces linear in $\dot{q}$, such as linear viscous friction, the techniques used to show that Newmark trajectories are shadowed by variational trajectories still apply, at least for the case $\beta=1 / 4$ and $\gamma=1 / 2$. That is, trajectories of the average acceleration Newmark with a linear viscous term added by setting $a_{k}=M^{-1}\left[-\nabla V\left(q_{k}\right)-\gamma \dot{q}\right]$ are shadowed, in the precise sense, by trajectories of the variational Lagrange-d'Alembert integrator (5.4) with $F(\dot{q})=-\gamma \dot{q}$.

Considerably greater insight may be achieved, however, by the realization that the technique of Suris used in Section 3.4 can be extended to the forced case by the appropriate choice of discrete force functions, showing that New-

mark with forcing is indeed a variational algorithm in the sense of the discrete Lagrange-d'Alembert principle.

The Newmark algorithm for a mechanical system with external forces $F(q, \dot{q})$ is given by

$$
\begin{aligned}
& q_{k+1}=q_{k}+h \dot{q}_{k}+\frac{h^{2}}{2}\left[(1-2 \beta) a_{k}+2 \beta a_{k+1}\right] \\
& \dot{q}_{k+1}=\dot{q}_{k}+h\left[(1-\gamma) a_{k}+\gamma a_{k+1}\right],
\end{aligned}
$$

where we now included forces in the acceleration terms to give

$$
a_{k}=M^{-1}\left[-\nabla V\left(q_{k}\right)+F\left(q_{k}, \dot{q_{k}}\right)\right] .
$$

The implicit function theorem can be used to check that (for $h$ sufficiently small) the Newmark equations (5.5), (5.6) and (5.7) implicitly define a mapping $\left(q_{k}, q_{k+1}\right) \mapsto\left(\dot{q}_{k}, \dot{q}_{k+1}\right)$. This allows us to replace the force evaluations in 
(5.7) with the expressions $F_{N M}^{\beta-}$ and $F_{N M}^{\beta+}$ defined by

$$
\begin{aligned}
& F_{N M}^{\beta-}\left(q_{k}, q_{k+1}\right)=F\left(q_{k}, \dot{q}_{k}\right) \\
& F_{N M}^{\beta+}\left(q_{k}, q_{k+1}\right)=F\left(q_{k+1}, \dot{q}_{k+1}\right),
\end{aligned}
$$

where $\dot{q}_{k}$ and $\dot{q}_{k+1}$ are given by the implicit mapping defined by the Newmark equations.

Having made this force substitution now allows us to write Newmark with forcing as an update of configuration points, in the same way as Section 3.1. This yields

$$
\begin{aligned}
\frac{1}{h}\left(q_{k+2}\right. & \left.-2 q_{k+1}+q_{k}\right) \\
+ & \beta h M^{-1} \nabla V\left(q_{k+2}\right)-(2 \beta-1) h M^{-1} \nabla V\left(q_{k+1}\right)+\beta h M^{-1} \nabla V\left(q_{k}\right) \\
& -\beta h M^{-1} F_{N M}^{\beta+}\left(q_{k+1}, q_{k+2}\right)+\frac{1}{2}(2 \beta-1) h M^{-1} F_{N M}^{\beta-}\left(q_{k+1}, q_{k+2}\right) \\
& +\frac{1}{2}(2 \beta-1) h M^{-1} F_{N M}^{\beta+}\left(q_{k}, q_{k+1}\right)-\beta h M^{-1} F_{N M}^{\beta-}\left(q_{k}, q_{k+1}\right)=0
\end{aligned}
$$

We will now establish that this algorithm can be derived as the forced discrete Lagrange-d'Alembert equations for the appropriate choice of discrete Lagrangian and discrete forces.

Theorem 5.1. The Newmark method with $\gamma=1 / 2$ and any $\beta$ acting on a forced system is the forced discrete Euler-Lagrange equations for the discrete Lagrangian $L_{d}^{\beta}$ and discrete forces $F_{d}^{\beta-}$ and $F_{d}^{\beta+}$ defined by

$$
\begin{aligned}
L_{d}^{\beta}\left(q_{0}, q_{1}\right) & =h \frac{1}{2}\left(\frac{\eta^{\beta}\left(q_{1}\right)-\eta^{\beta}\left(q_{0}\right)}{h}\right)^{T} M\left(\frac{\eta^{\beta}\left(q_{1}\right)-\eta^{\beta}\left(q_{0}\right)}{h}\right)-h \tilde{V}\left(\eta^{\beta}\left(q_{0}\right)\right) \\
F_{d}^{\beta-}\left(q_{0}, q_{1}\right) & =h \frac{1}{2}\left[(1-2 \beta) F_{N M}^{\beta-}\left(q_{0}, q_{1}\right)+2 \beta F_{N M}^{\beta+}\left(q_{0}, q_{1}\right)\right] D \eta^{\beta}\left(q_{0}\right) \\
F_{d}^{\beta+}\left(q_{0}, q_{1}\right) & =h \frac{1}{2}\left[2 \beta F_{N M}^{\beta-}\left(q_{0}, q_{1}\right)+(1-2 \beta) F_{N M}^{\beta+}\left(q_{0}, q_{1}\right)\right] D \eta^{\beta}\left(q_{1}\right)
\end{aligned}
$$

where $\eta^{\beta}\left(q_{k}\right)=q_{k}-h^{2} \beta M^{-1} \nabla V\left(q_{k}\right), \tilde{V}$ is defined so that $\nabla \tilde{V}\left(\eta^{\beta}\left(q_{k}\right)\right)=$ $\nabla V\left(q_{k}\right)$ for all $q_{k}$ and the functions $F_{N M}^{\beta-}$ and $F_{N M}^{\beta+}$ are as defined in (5.8) and $(5.9)$.

Proof. We begin by noting that the discrete Lagrangian $L_{d}^{\beta}$, the mapping $\eta^{\beta}$ and the modified potential $\tilde{V}$ are all identical to those used in Theorem 3.5 for the unforced conservative case. We therefore know that $\tilde{V}$ and thus $L_{d}^{\beta}$ are well-defined, and we have already established above that $F_{N M}^{\beta-}$ and $F_{N M}^{\beta+}$ 
exist, and hence so do $F_{d}^{\beta-}$ and $F_{d}^{\beta+}$. It only remains to check that the forced discrete Euler-Lagrange equations for (5.11)-(5.13) give the position-update form (5.10) of forced Newmark.

This is a simple matter of evaluating (5.3), multiplying on the right by the inverse of $D \eta^{\beta}\left(q_{1}\right)$ and substituting the expression for $\eta^{\beta}$. Rearranging then immediately gives (5.10), as claimed.

The fact that Newmark with $\gamma=1 / 2$ is a second order accurate discrete Lagrange-d'Alembert integrator implies that its performance should be similar to that of the algorithm (5.4). As we will see in the numerical tests in Section 6 , this is indeed the case.

\subsection{A Two-Step Variational Principle for Friction}

Next, we explore a two step algorithm for problems with friction where the two steps are designed to split the algorithm into conservative and dissipative parts. We will stick with the case of a Lagrangian of the form kinetic minus potential energies for simplicity. The second step is based on an interesting minimization principle.

We believe this algorithm and its companion algorithm which uses an explicit-implicit split, which are based on optimization methods, may be useful in certain large problems where the computational savings using optimization techniques can be employed. However, in the present paper we consider only simple, low dimensional numerical examples. These two step algorithms are employed in the work on collisions (see Kane, Repetto, Ortiz and Marsden [1999] and Kane, Marsden, Ortiz and Pandolfi [1999]).

Given $\left(q_{0}, q_{1}\right)$, we first compute the point $q_{2}^{\text {pred }}$ according to the discrete Euler-Lagrange equations for a given $L_{d}$. For example, with the discrete Lagrangian $L_{d}^{\alpha}$ with $\alpha=1 / 2, q_{2}^{\text {pred }}$ satisfies

$$
M\left[\left(\frac{q_{2}^{\text {pred }}-2 q_{1}+q_{0}}{h^{2}}\right)\right]+\frac{1}{2}\left[V^{\prime}\left(\frac{q_{0}+q_{1}}{2}\right)+V^{\prime}\left(\frac{q_{1}+q_{2}^{\text {pred }}}{2}\right)\right]=0 .
$$

Of course, as we showed in the first part of the paper, suitably interpreted, this step also includes the Newmark algorithm. Then we follow this with a second (dissipative) step, which consists of minimizing the discrete kinetic energy plus the dissipative potential with respect to the last endpoint.

That is, we extremize the following expression with respect to the final endpoint $q_{2}$ :

$$
\frac{1}{2}\left(\frac{q_{2}-q_{2}^{\text {pred }}}{h}\right)^{T} M\left(\frac{q_{2}-q_{2}^{\text {pred }}}{h}\right)+h \varphi\left(\frac{q_{2}-q_{1}}{h}\right) .
$$


In other words, the equation satisfied by $q_{2}$ is given by

$$
M\left(\frac{q_{2}-q_{2}^{\text {pred }}}{h^{2}}\right)+\varphi^{\prime}\left(\frac{q_{2}-q_{1}}{h}\right)=0 .
$$

Adding (5.14) and (5.16), we see that the term involving $q_{2}^{\text {pred }}$ cancels and we get

$$
\begin{aligned}
M[ & \left.\left(\frac{q_{2}-2 q_{1}+q_{0}}{h^{2}}\right)\right] \\
& +\frac{1}{2}\left[V^{\prime}\left(\frac{q_{0}+q_{1}}{2}\right)+V^{\prime}\left(\frac{q_{1}+q_{2}^{\text {pred }}}{2}\right)\right] \\
& +\varphi^{\prime}\left(\frac{q_{2}-q_{1}}{h}\right)=0,
\end{aligned}
$$

which is consistent with the original equations.

We summarize the result in the following

Theorem 5.2. For a Lagrangian of the form kinetic minus potential energy, the following two-step algorithm is consistent with the equations of motion with dissipative forces derived from a dissipative potential $\varphi$ :

Step 1 Map $\left(q_{n-1}, q_{n}\right)$ to $\left(q_{n}, q_{n+1}^{\text {pred }}\right)$ by means of the discrete Euler-Lagrange equations for a choice of discrete Lagrangian $L_{d}$

Step $2 \operatorname{Map}\left(q_{n}, q_{n+1}^{\text {pred }}\right)$ to $\left(q_{n}, q_{n+1}\right)$ by extremizing

$$
K_{d}\left(q_{n+1}, q_{n+1}^{\text {pred }}\right)+h \varphi\left(\frac{q_{n+1}-q_{n}}{h}\right),
$$

where $K_{d}$ is the discrete kinetic energy, with respect to the final endpoint $q_{n+1}$.

While this algorithm is not literally a product formula (in the sense of, eg, Chorin, Hughes, Marsden and McCracken [1978]), it has some of the same spirit. It would be of interest of course to see to what extent one can prove things about the behavior of the symplectic form and the energy.

\subsection{Minimization Structure of Newmark with Friction.}

As we explained in $§ 3.6$, one can write the Newmark algorithm with external forces using an explicit-implicit split, where all of the implicit part of the algorithm is bundled with a minimization step. We just note that compared 
to other possible formulations of Newmark, the minimization scheme has an additional parameter $\sigma$ used for interpolation in the force evaluations, so it may depart slightly from other Newmark schemes with external forcing. In the Newmark simulations below, we mean the ones generated by the scheme given in $\S 3.6$ with the computed acceleration initialized to the true acceleration.

One of the advantages of the optimization approach in the scheme here as well as the one in the preceding section is that it extends in a natural way to problems with Coulomb friction, where one has to also do an optimization over the friction cone; however, the basic structure of the scheme remains intact. As we have remarked, this extension of the present method combined with our work on collisions is discussed in Kane, Marsden, Ortiz and Pandolfi [1999].

\section{$6 \quad$ Numerical Tests for Dissipative Systems}

\subsection{Example System}

To demonstrate the behavior of the algorithms developed in the previous section we consider the same non-linear oscillator used in $\S 4$ with a small amount of linear viscous dissipation. In the notation used in the previous section, we use the dissipative potential given by

$$
\varphi(\dot{q})=\frac{10^{-3}}{2} \dot{q}^{T} \dot{q}
$$

which corresponds to a force of

$$
F(\dot{q})=-10^{-3} \dot{q}
$$

We have chosen a weakly dissipative system to highlight the advantages of the Newmark, variational and two-step methods. If the dissipation is too high, then all trajectories quickly decay to zero energy and it is difficult to distinguish any differences between the integrators.

As for the conservative example presented previously, we concentrate here on the accuracy of the integrators at estimating the energy and momentum evolution. The dissipative nature of the system implies that both quantities should decrease, so the test becomes the correct estimation of the overall decay of energy and momentum.

\subsection{Tested Algorithms}

We present results for the same methods used on the conservative system, except that here we use the two extensions to the variational algorithm for dissipative systems, namely the discrete Lagrange-d'Alembert method and the two-step minimum work method. 
- Implicit Newmark: Newmark with $\beta=1 / 4, \gamma=1 / 2$

- Explicit Newmark: Newmark with $\beta=0, \gamma=1 / 2$

- Variational: $L_{d}^{\mathrm{sym}, \alpha}, F_{d}^{\mathrm{sym}, \alpha}$ discrete Lagrange-d'Alembert method with $\alpha=1 / 2$

- Two-step: Two-step minimum work method

- Runge-Kutta: 4th order, fixed time-step

- Benchmark: MATLAB 5.3 ODE113 (Predictor-corrector)

The integration parameters, such as step size and method of energy evaluation, are all identical to those used in Section 4 for the numerical tests in the conservative case.

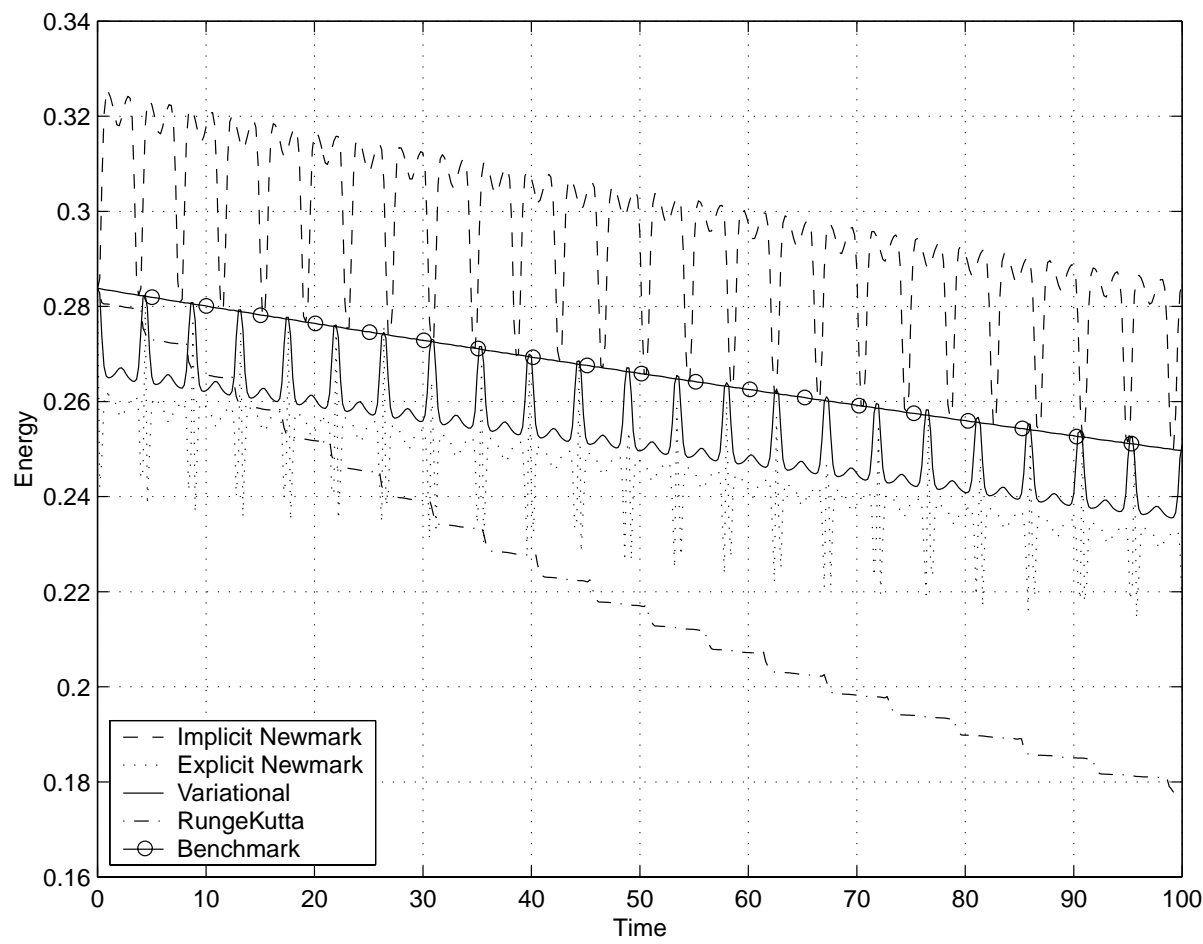

Figure 6.1: Energy behavior of integrators for a dissipative system. The variational integrators accurately simulate energy decay, unlike standard methods such as Runge-Kutta.

\subsection{Results}

Two tests are presented here, both on the same system. In Figure 6.1 the simulation is run for the same time length as was the conservative system in 


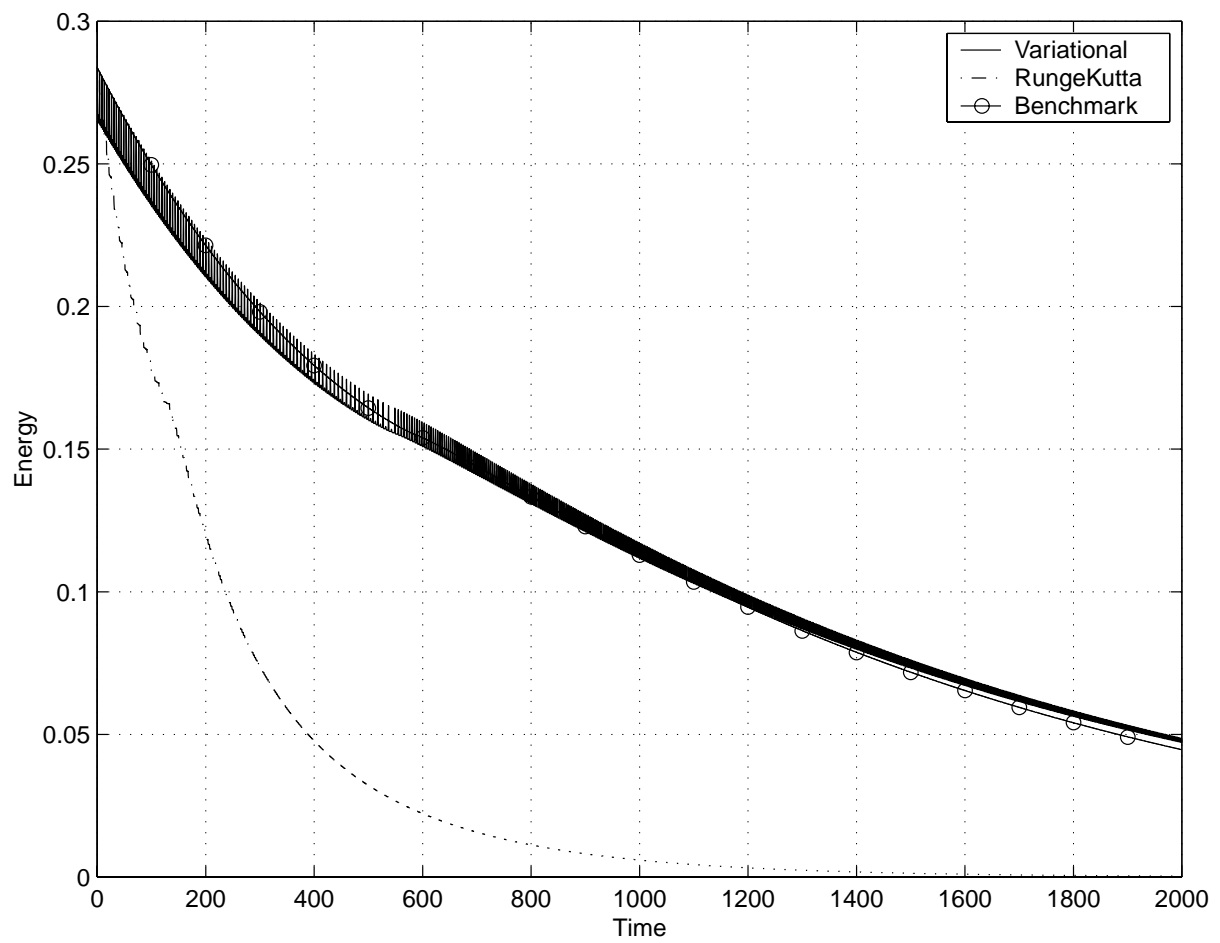

Figure 6.2: Energy behavior of integrators for a dissipative system. The variational integrators accurately simulate energy decay, unlike standard methods such as Runge-Kutta.

Section 4. As a more dramatic demonstration we also run the system for a very much longer time, as given in Figure 6.2. In both cases we plot only the energy decay. We do not give the corresponding momentum plots, as they are qualitatively similar to the energy.

For the example system, the discrete Lagrange-d'Alembert variational method and the two-step variational method give results which are almost indistinguishable. For this reason only a single 'Variational' trajectory is plotted in Figures 6.1 and 6.2. This should be taken as representative of both the discrete Lagrange-d'Alembert variational method and the two-step variational method. The crucial aspect of both of these algorithms is their variational nature.

From these results it is clear that the Newmark method, the discrete Lagrange-d'Alembert method and the two-step minimum work method all correctly capture the energy decay, unlike traditional methods such as RungeKutta. When the simulation is continued for longer times, this good behavior continues, although there are slight deviations at very large times.

The particularly impressive aspect of the energy decay predictions of the variational integrators is that they are only low order methods, unlike the fourth order accurate Runge-Kutta. This is a clear demonstration of the fact 
that traditional measures of integrator accuracy, such as local truncation error, are not necessarily appropriate when discussing variational or symplectic schemes, as they often perform far better than expected.

As shown previously, the Newmark algorithm is variational, and so we expect the good energy behavior seen here. For this reason we have omitted the Newmark method from the longer time simulation, in order to make the results clearer. In that case Newmark performs similarly to the other variational methods.

The variational integrators for dissipative systems are expected to be particularly useful for systems which are nearly conservative, such as mechanical systems with weak dissipation or weak forcing. This is due to the fact that they perform very well on the main conservative part of the system, the area where traditional integration schemes introduce most of the error.

Although the example chosen here is very simple, it captures the important aspects of the numerical behavior of the variational algorithms. The performance of the algorithms on high degree of freedom systems, such as finite element discretizations, is of considerable interest.

\section{Conclusions and Future Work}

We conclude with some general comments and possible directions for future work.

Higher-Order Integrators. In future work we plan to develop higher order integrators based on, for example, more accurate approximations to the action integral. This is closely related to the technique of forming good approximations to the Hamilton-Jacobi equation, as in Channell and Scovel [1990], but we believe that one can sometimes be better off using Jacobi's solution to the Hamilton-Jacobi equation (the integral of the Lagrangian along a solution to the Euler-Lagrange equations) and approximating this integral.

Nonlinear Analysis of Newmark. It is quite possible that the techniques of this paper can be used to give a nonlinear analysis showing the good long time performance of the Newmark algorithm. At the moment, most of the analysis is that of error analysis type and for linear systems.

Time Adaptive Algorithms. As shown in Kane, Marsden and Ortiz [1999], one can achieve conservation of energy in addition to conserving the symplectic structure (in an appropriate spacetime sense) and momentum by using time adaptive techniques. It would be of interest to explore the numerical implications of this further in the context of the present paper. 
Collisions. In Kane, Repetto, Ortiz and Marsden [1999] algorithms for collision problems are developed. In fact, those algorithms are consistent with those presented in this paper. The paper Kane, Marsden, Ortiz and Pandolfi [1999] explores the addition of friction to these algorithms.

Multisymplectic Integrators. Another area of interest is the development of multisymplectic integrators for PDE's following Marsden, Patrick and Shkoller [1998]. It would be of interest to explore these integrators using Newmark methods coupled with finite element techniques as well as with the addition of friction or forcing.

\section{Acknowledgments.}

We thank Petr Krysl and Sanjay Lall for helpful comments and inspiration, and Yuri Suris for suggesting how Newmark might be directly expressed as a variational algorithm. We are also grateful to the referees for their constructive and helpful comments.

\section{References}

Armero, F. and J.C. Simo [1993] A-Priori Stability Estimates and Unconditionally Stable Product Formula Algorithms for Non-Linear Coupled Thermoplasticity. Int. J of Plasticity, 9, 149-182.

Armero, F. and J.C. Simo [1996] Long-Term Dissipativity of Time-Stepping Algorithms for an Abstract Evolution Equation with Applications to the Incompressible MHD and Navier-Stokes Equations. Comp. Meth. Appl. Mech. Eng. 131, 41-90.

Armero, F. and J.C. Simo [1996] Formulation of a new class of fraction-step methods for the incompressible MHD equations that retains the long-term dissipativity of the continuum dynamical system, Fields Institute Comm. 10, 1-23.

Bobenko, A. I., B. Lorbeer and Yu. B. Suris [1998] Integrable discretizations of the Euler top. J. Math. Phys. 39, 6668-6683.

Bobenko, A.I. and Yu. B. Suris [1998] Discrete time Lagrangian mechanics on Lie groups, with an application to the Lagrange top, preprint.

Channell, P. and C. Scovel [1990] Symplectic integration of Hamiltonian systems, Nonlinearity 3, 231-259.

Chorin, A.J, T.J.R. Hughes, J.E. Marsden, and M. McCracken [1978] Product Formulas and Numerical Algorithms, Comm. Pure Appl. Math. 31, 205-256.

Chung, J. and G.M. Hulbert [1993] A Time Integration Algorithm For Structural Dynamics with improved numerical dissipation - the generalized- $\alpha$ method Journal Of Applied Mechanics-Transactions Of The ASME60, 371-375.

Ge, Z. and J.E. Marsden [1988] Lie-Poisson integrators and Lie-Poisson Hamilton-Jacobi theory, Phys. Lett. A 133, 134-139. 
Geradin, M. and D. Rixen [1993] Thèorie des vibrations-Application a la dynamique des structures, Mason.

Gonzalez, O. [1996]. Time integration and discrete Hamiltonian systems. J. Nonlinear Sci. 6, 449-468.

Gonzalez, O and Simo, JC [1996] On the stability of symplectic and energy-momentum algorithms for non-linear Hamiltonian systems with symmetry, Comp. Meth. App. Mech. Eng. 134(3-4), 197-222.

Hairer, E. and C. Lubich [1997] The life-span of backward error analysis for numerical integrators. Numer. Math. 76, 441-462.

Hughes, T. J. R. [1987] The finite element method. Linear static and dynamic finite element analysis, Prentice-Hall.

Kane, C, J.E. Marsden, and M. Ortiz [1999] Symplectic energy momentum integrators, $J$. Math. Phys. 40, 3353-3371.

Kane, C, J.E. Marsden, M. Ortiz and A. Pandolfi [1999] Time-discretized variational formulation of nonsmooth frictional contact (in preparation).

Kane, C, E.A. Repetto, M. Ortiz and J.E. Marsden [1999] Finite element analysis of nonsmooth contact Computer Meth. in Appl. Mech. and Eng., (to appear).

Leimkuhler, B. and S. Reich [1994]. Symplectic integration of constrained Hamiltonian systems. Math. Comp. 63, 589-605

MacKay, R.S. [1992] Some aspects of the dynamics and numerics of Hamiltonian systems, in The Dynamics of Numerics and the Numerics of Dynamics (ed. D.S. Broomhead and A. Iserles), Oxford University Press, 137-193

Marsden, J. E., G. W. Patrick, and W. F. Shadwick [1996] Integration Algorithms and Classical Mechanics. Fields Institute Communications, 10, Am. Math. Society.

Marsden, J. E., G. W. Patrick, and S. Shkoller [1998] Multisymplectic Geometry, Variational Integrators, and Nonlinear PDEs Comm. Math. Phys. 199, 351-395.

Marsden, J.E., S. Pekarsky and S. Shkoller [1999a] The discrete Euler-Poincaré equation and reduction. preprint.

Marsden, J.E., S. Pekarsky and S. Shkoller [1999b] Symmetry Reduction of Discrete Lagrangian Mechanics on Lie groups preprint.

McLachlan, R. I. and C. Scovel [1996]. A survey of open problems in symplectic integration. Fields Institute Communications, 10, 151-180.

Moser, J. and A.P. Veselov [1991] Discrete versions of some classical integrable systems and factorization of matrix polynomials. Comm. Math. Phys. 139, 217-243.

Newmark N.M. [1959] A Method of Computation for Structural Dynamics, ASCE J. of the Engineering Mechanics Division, 67-94.

Ortiz, M. [1986] A note on energy conservation and stability of nonlinear time-stepping algorithms. Computers and Structures, 24, 167-168.

Ortiz, M. and L. Stainier [1999]. The variational formulation of viscoplastic constitutive updates. Computer Methods in Appl. Mech. and Eng., 171, 419-444. 
Radovitzky, R. and M. Ortiz [1999]. Error estimation and adaptive meshing in strongly nonlinear dynamic problems. Computer Methods in Appl. Mech. and Eng., 172, 203-240.

Reich, S. [1994]. Momentum preserving symplectic integrators. Physica D, 76, 375-383.

Reich, S. [1999]. Backward error analysis for numerical integrators. SIAM J. Numer. Anal. 36, 1549-1570.

Reich, S. and T. Bridges [1999] Multi-Symplectic Integrators: numerical schemes for Hamiltonian PDEs that conserve symplecticity (preprint).

Sanz-Serna, J. M. and M. Calvo [1994] Numerical Hamiltonian Problems. Chapman and Hall, London.

Simo, J.C.and O. Gonzalez. [1993], Assessment of Energy-Momentum and Symplectic Schemes for Stiff Dynamical Systems. Proc. ASME Winter Annual Meeting, New Orleans, Dec. 1993.

Simo, J.C. and N. Tarnow [1992] The discrete energy momentum method. Conserving algorithms for nonlinear elastodynamics, ZAMP 43, 757-792.

Simo, J.C., N. Tarnow, and K.K. Wong [1992]. Exact energy-momentum conserving algorithms and symplectic schemes for nonlinear dynamics Comp. Meth. Appl. Mech. Eng. $10063-116$.

Veselov, A.P. [1988] Integrable discrete-time systems and difference operators. Funct. An. and Appl. 22, 83-94.

Wendlandt, J.M. and J.E. Marsden [1997] Mechanical integrators derived from a discrete variational principle, Physica D 106, 223-246. 